Paper

\title{
Nonlinearity in simple phenomenological model for activity-dependent responses of target-cell specific synapses
}

\author{
Hideyuki Kato ${ }^{1 \text { a) }}$ \\ ${ }^{1}$ Faculty of Science and Technology, Oita University, \\ 700 Dannoharu, Oita-city, 870-1192, Japan \\ a)h-kato@oita-u.ac.jp
}

Received December 23, 2020; Revised March 20, 2021; Published July 1, 2021

\begin{abstract}
Synaptic responses in activity-dependent manners are thought to play crucial roles in neuronal information processing and are called short-term synaptic plasticity. Typically, two major types of short-term plasticity are observed in the cortical or the hippocampal synapses: facilitation and depression. Although these synaptic responses are reproducible in a canonical phenomenological model, synaptic responses are diverse and target-cell specific, and then the phenomenological model does not cover synaptic responses such as facilitation growth and facilitation-depression. In contrast, while detailed models of short-term plasticity can trace the two synaptic responses, its computational costs are too heavy to simulate large-scale neuronal networks whose simulations are necessary to clarify functional roles and mechanisms of information processing in the cortical or the hippocampal fine-scale circuits. To understand them, the realization of the synaptic responses by a simple STP model is an important issue. In the current study, it is demonstrated a small extension of the canonical phenomenological model makes it possible to realize the synaptic responses of the facilitation growth and the facilitationdepression. Besides, the current study conducts basic analyses of responses in the synapses to both regular and random action potential trains and provides results of the analyses because the nature of the synapses is not clarified yet.
\end{abstract}

Key Words: activity-dependent synaptic responses, short-term plasticity, information processing, target-cell specific synapses

\section{Introduction}

Neurons in the brain interact with one another by sending and receiving signals called spikes or action potentials (APs) via chemical synapses. Electrophysiological studies of the cortical and/or the hippocampal neurons often observe a phenomenon in which presynaptic AP effects on postsynaptic potential (PSP) of various types of synapses dynamically change according to a history of presynaptic activity [1-6]. The phenomenon, which is termed short-term synaptic plasticity (STP), is divided into two categories: depression and facilitation [7-9]. In the depression, the second presynaptic AP efficacy is smaller than the first one. Contrary, the response evoked by the second presynaptic AP 
is enhanced in the facilitation. The changes of synaptic effects last in hundreds of millisecond to seconds.

It is suggested STP plays an important role for sustaining internal states [10-12] and neuronal information processing $[13,14]$. Several theoretical studies suggest various functional roles of this phenomenon, such as low-pass, band-pass and high-pass filtering effects $[15,16]$, insensitivity of a postsynaptic neuron to absolute intensity of presynaptic activity [17,18], making presynaptic AP trains decorrelated [19], optimizing synaptic information transmission [20], an optimal presynaptic AP estimator [21], sustaining working memory [22], and so on. STP among synapses, however, are highly heterogeneous [23, 24], and then its primitive functions are still unrevealed.

Tsodyks-Markram (TM) model covers and realizes the typical synaptic responses of the depression and the facilitation in STP [4]. A form of the canonical phenomenological model of STP is frequencydependent of presynaptic APs and consists of two variables with three parameters. The model is described as

$$
\begin{aligned}
& \frac{\mathrm{d} x}{\mathrm{~d} t}=\frac{1-x}{\tau_{d}}-u^{+} x^{-} \sum_{n} \delta\left(t-t_{n}^{\mathrm{sp}}\right), \\
& \frac{\mathrm{d} u}{\mathrm{~d} t}=-\frac{u}{\tau_{f}}+U\left(1-u^{-}\right) \sum_{n} \delta\left(t-t_{n}^{\mathrm{sp}}\right),
\end{aligned}
$$

where $x$ is the fraction of available remaining resources after neurotransmitter releases and $u$ is the neurotransmitter release probability [25]. The parameters $\tau_{d}$ and $\tau_{f}$ are respectively a time constant of the variables $x$ and $u$ and typical values of the parameters are in tens to thousands of milliseconds. The superscripts of - and + in Eqs. (1) and (2) respectively represent a value of the variables just before and after updates triggered by presynaptic APs. Namely, $x^{-}=\lim _{t \rightarrow t_{n}^{\mathrm{s}}-0} x(t), x^{+}=\lim _{t \rightarrow t_{n}^{\mathrm{sp}}+0} x(t)$, $u^{-}=\lim _{t \rightarrow t_{n}^{\mathrm{sP}}-0} u(t)$, and $u^{+}=\lim _{t \rightarrow t_{n}^{\mathrm{sp}}+0} u(t)$, respectively. The time $t_{n}^{\mathrm{sp}}$ was the $n$th presynaptic AP arrival in Eqs. (1) and (2). Synapses tend to favor facilitation for a small value of $U$ bounding in a range of $(0,1]$, while a large value of $U$ makes synapses depression-favor. Mismatches of the parameters $\tau_{d}$ and $\tau_{f}$ determining the time scale of the two variables generate depression or facilitation effects on synaptic responses. Postsynaptic current induced by an STP synapse is written as $I(t)=g(E-V)$ where $E$ is reversal potential, $V$ is postsynaptic membrane potential in millivolts and $g$, whose kinetics is

$$
\frac{\mathrm{d} g}{\mathrm{~d} t}=-\frac{g}{\tau_{\mathrm{syn}}}+\sum_{n} \bar{g} x^{-} u^{+} \delta\left(t-t_{n}^{\mathrm{sp}}\right),
$$

where $\bar{g}$ is the synaptic potency, $\tau_{\text {syn }}$ is the synaptic time constant in the milliseconds. This model, however, cannot reproduce some types of synaptic responses observed in electrophysiological studies because of its linearity.

Empirical studies have shown synaptic responses to the presence of regular AP trains are diverse and target-cell specific. For example, the amplitude of synaptic responses grows supralinearly. This is termed facilitation growth (FG). The FG is found in proximal muscle fibers of crayfish [26-32] and in excitatory synapses on low-threshold spiking neurons, which are inhibitory interneurons, in the layer 4 rat barrel cortex [23]. The model introducing an assumption of the existence of immobile $\mathrm{Ca}^{2+}$ buffers well mimics the FG in the synapses. Another example is that hippocampal CA3 to CA1 Shaffer collateral synapses exhibit large facilitation at the beginning of stimulations and depress after the facilitation [16]. This type of STP is termed as facilitation-depression (FD). A model assuming the equilibrium occupancy of the release site by a calcium-bound molecule accounts for FD behavior. In contrast to the phenomenological STP model, these biophysically detailed STP models have an ability to trace the two types of synaptic responses in Refs. [16, 26-30,32]. These models are meaningful to unveil structures and mechanisms of presynapic terminals, but the models are inefficient for large-scale neural network simulations due to their complexity. An FG- and an FDreproducible but computationally efficient STP model is inevitable for understanding functional roles and/or mechanisms of information processing in the cortical and/or hippocampal fine-scale circuits. 
To realize the above terget-specific synaptic responses with keeping the simplicity of an STP model in the current study, I propose a phenomenological STP model by introducing a nonlinear function into the second term of Eq. (2) in Tsodyks-Markram STP model. The proposed model can well mimic both FG and FD reported in Refs. [16,23] because of its nonlinearity. Besides, the current study also provides results of some analayses on synaptic responses of FG and FD synapses to regular and random AP trains.

\section{Materials and methods}

\subsection{Augmentation of phenomenological STP model}

A proposal in the current study was an augmentation of TM model defined in Eqs. (1) and (2). Although the typical synaptic responses in the cortex and/or the hippocampus were reproducible in the canonical model, the model could not mimic responses of some specific synapses, that is, FG and FD reported in Refs. $[16,23,26]$. The limitation of TM model derived from its linearity. Because of its linearity, the second term of Eq. (2) linearly decreased as $u$ increased. This constrained the canonical STP model to generate the two types of specific responses. Therefore, if the STP model had some types of nonlinearity, the synaptic responses might be reproducible in the model.

Based on this idea, a nonlinear function of $f(u)$ was multiplied into the second term of Eq. (2). The proposed model then had the following form:

$$
\frac{\mathrm{d} u}{\mathrm{~d} t}=-\frac{u}{\tau_{f}}+U f\left(u^{-}\right)\left(1-u^{-}\right) \sum_{n} \delta\left(t-t_{n}^{\mathrm{sp}}\right) .
$$

In the current study, $f(u)=U^{-0.5}\left\{a-(\alpha u+b)^{-1}\right\}$ was adopted (Fig. 1(A)) as the multiplied nonlinear function. Here, it should be noted any types of nonlinear functions were possible to be chosen if $f(u)$ monotonically increased. The choice of $f(u)$, however, allowed the computational cost of the augmented model to be reduced (See also below). Consequently, the kinetics of $u$ of the augmented STP model was described by

$$
\frac{\mathrm{d} u}{\mathrm{~d} t}=-\frac{u}{\tau_{f}}+\sqrt{U}\left(a-\frac{1}{\alpha u^{-}+b}\right)\left(1-u^{-}\right) \sum_{n} \delta\left(t-t_{n}^{\mathrm{sp}}\right),
$$

where $a$ and $b$ were constant values yielded from the condition satisfying the $f(u)$ always passed through $(0,1)$ and $(1,1 / \sqrt{U})$. The constant values $a$ and $b$, that were determined by only the parameters $U$ and $\alpha$, had the following forms:

$$
\begin{aligned}
& a=\sqrt{U}+\frac{1-\sqrt{U}}{2}\left\{1+\sqrt{1+\frac{4}{\alpha(1-\sqrt{U})}}\right\}, \\
& b=\frac{\alpha}{2}\left\{\sqrt{1+\frac{4}{\alpha(1-\sqrt{U})}}-1\right\} .
\end{aligned}
$$

The parameter $\alpha(>0)$ controlled the bending degree of $f(u)$ (Fig. 1(A)). As seen in Fig. 1(B), the resultant update function in Eq. (5) was convex upward. The smaller $\alpha$ made $f(u)$ closer to the original linear function in TM model, indicating the augmented STP model was almost equivalent to TM model when $\alpha$ was small.

To observe effect of the augmented STP model on postsynaptic responses evoked by presynaptic APs, a leaky integrator simulated the responses and its formulation was the following:

$$
C_{\mathrm{m}} \frac{\mathrm{d} V}{\mathrm{~d} t}=g_{l}\left(V_{\text {hold }}-V\right)+I(t),
$$

where $V$ was the membrane potential, $C_{\mathrm{m}}$ was the membrane capacitance, $V_{\text {hold }}$ was the holding potential in the absence of presynaptic APs, $g_{l}$ was the leak conductance. $I(t)=g(V-E)$ was the presynaptic input. In all simulations of the current study, the parameters in Eqs. (3) and (8) were 
A

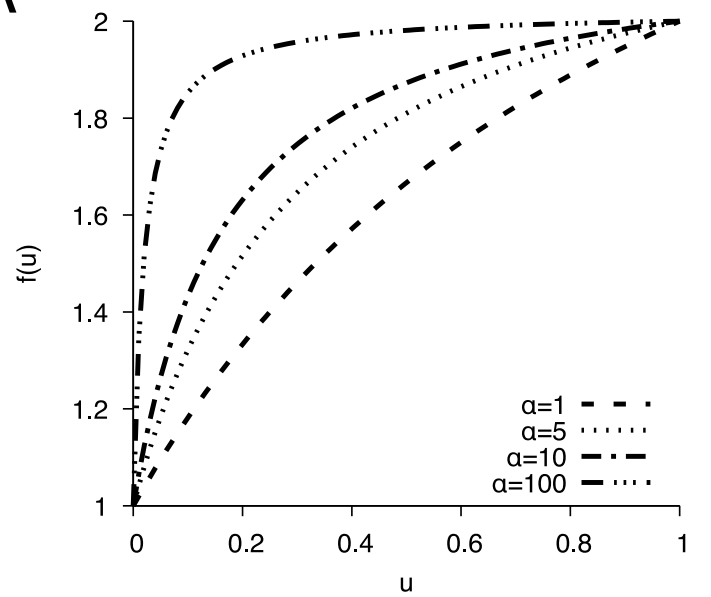

B

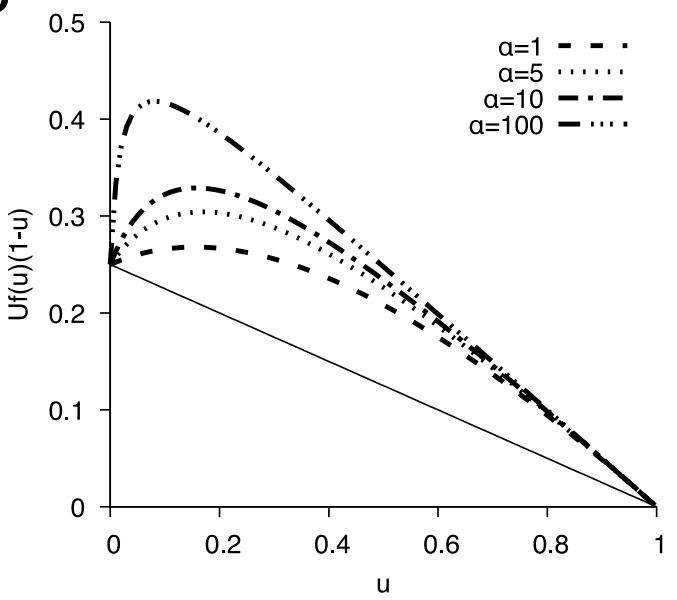

Fig. 1. Examples of (A) the nonlinear function $f(u)$ and $(\mathrm{B})$ the resultant presynaptic AP effects in Eq. (5). The parameter $U$ is set to 0.25. The solid line is the update function in the canonical model of Eq. (2).

set as $\tau_{\mathrm{syn}}=2 \mathrm{~ms}, \tau_{\mathrm{m}}=C_{\mathrm{m}} / g_{l}=10 \mathrm{~ms}, V_{\text {hold }}=-62 \mathrm{mV}$, and $g_{l}=20 \mathrm{nS}$. Besides, throughout this paper, $E=0$ was used, which was assumed all synaptic responses were excitatory postsynaptic potentials (EPSPs). The parameters of all types of synapses were set as the EPSP evoked by the first presynaptic AP became $0.5 \mathrm{mV}$. The time resolution of all numerical simulations in the current study was $0.1 \mathrm{~ms}$. The reason why a firing threshold was not introduced was to understand pure effect of the augmented STP model on postsynaptic responses.

\subsection{Data for analyses}

In the current study, synaptic responses to both regular and random presynaptic AP trains were numerically analyzed. In the case of the regular ones, the numerical simulations were continued until the difference between the $(n-1)$ th and the $n$th response was less than $10^{-6}$ to evaluate the steady-state values for presynaptic AP trains with individual rates.

In the analysis on synaptic responses to random presynaptic AP trains, the AP trains were generated by Poisson process with the mean firing rate of $\lambda(\mathrm{sp} / \mathrm{s})$. The synaptic responses to the random trains never settled down into a steady-state value in contrast to those to the regular trains. For the characterization of the synaptic responses, 105,000 synaptic responses were collected and the first 100 responses were ignored as the transient responses in the analysis of the mean response and the creation of tuning curves. In the mean response analysis, the first 100,000 synaptic responses were subtracted to obtain the mean value after the elimination of the 100 synaptic responses. For the creation of tuning curves (see also below), all the synaptic responses for the ISIs larger then eight-fold of the mean ISI were also eliminated from the data after the 100 response elimination because these ISIs rarely occurred due to their low probabilities. By using the data after the procedure, the tuning curves were created from the first 100,000 synaptic responses. In information-theoretic analyses, there were no limitations of ISIs in the information-theoretic analysis (see also below). In the case of the information-theoretic analyses, numerical simulations generated 10,100 synaptic responses and the first 100 transient responses were removed from the generated responses. The mutual information was computed with the 10,000 synaptic responses. Also, 100 different realizations were averaged over for the estimation of mutual information.

\subsection{Theoretical predictions of steady-state value in synaptic response to regular- presynaptic-AP trains}

Even though the dynamics of the variables $u$ and $x$ were both written by the ordinary differential equation with the presynaptic-AP effects, from Eq. (3), it was clear the computation needed only the values of $u$ and $x$ at the moments of presynaptic AP arrivals. Then, iterative expressions of Eqs. (1) 
and (5) did not only make the model easier to analyze analytically but also was more efficient from a viewpoint of large-scale neural network simulations because solving the differential equations did not need. Here, let me define the values of $x^{-}$and $u^{+}$at the $(n+1)$ th presynaptic AP arrival as $x_{n+1}^{-}$ and $u_{n+1}^{+}$, namely, $x_{n+1}^{-}=\lim _{t \rightarrow t_{n+1}^{\mathrm{s}}-0} x(t)$ and $u_{n+1}^{+}=\lim _{t \rightarrow t_{n+1}^{\mathrm{sp}}+0} u(t)$. After the simple transformations, $x_{n+1}^{-}$and $u_{n+1}^{+}$could respectively be given as the followings:

$$
\begin{aligned}
& x_{n+1}^{-}=1-\left\{1-\left(1-u_{n}^{+}\right) x_{n}^{-}\right\} e^{-\frac{\Delta t_{n}}{\tau_{d}}}, \\
& u_{n+1}^{+}=u_{n}^{+} e^{-\frac{\Delta t_{n}}{\tau_{f}}}+\sqrt{U}\left(1-u_{n}^{+} e^{-\frac{\Delta t_{n}}{\tau_{f}}}\right)\left(a-\frac{1}{\alpha u_{n}^{+} e^{-\frac{\Delta t_{n}}{\tau_{f}}}+b}\right) .
\end{aligned}
$$

The above iterative expressions of $x^{-}$and $u^{+}$were satisfied for natural numbers $n$ when the followings were assumed: $x_{0}^{-}=1, u_{0}^{+}=0, \Delta t_{n}=t_{n+1}^{\mathrm{sp}}-t_{n}^{\mathrm{sp}}$, and $t_{0}^{\mathrm{sp}}=0$ which was a virtual presynaptic AP.

Next, the trajectory of $V$ after the $n$th presynaptic AP was yielded to obtain the amplitude of postsynaptic neuronal responses. First, $g(t)$ of Eq. (3) was considered to obtain the synaptic current $I(t)$ in Eq. (8). Let me define $g_{n}^{-}=\lim _{t \rightarrow t_{n}^{\mathrm{sP}}-0} g(t)$ and $g_{n}^{+}=\lim _{t \rightarrow t_{n}^{\mathrm{sp}}+0} g(t)$. Using these descriptions, the following equation could be obtained from Eq. (3).

$$
g(t)=g_{n}^{+} e^{-\frac{t-t_{n}^{\mathrm{sp}}}{\tau_{\mathrm{syn}}}}
$$

Further, the relation between $g_{n}^{+}$and $g_{n+1}^{+}$could be written as

$$
g_{n+1}^{+}=g_{n}^{+} e^{-\frac{\Delta t_{n}}{\tau_{\mathrm{syn}}}}+\bar{g} u_{n+1}^{+} x_{n+1}^{-} .
$$

where $g_{0}^{+}=0$ because from Eq. (3) $g_{n+1}^{-}$was increased by $\bar{g} u_{n+1}^{+} x_{n+1}^{-}$at the moment of the $(n+1)$ th presynaptic AP arrival. The approximation of $I(t)=g(E-V) \approx g\left(E-V_{\text {hold }}\right)$ allowed me to lead Eq. (8) to the following solution:

$$
V(t)=V_{\text {hold }}+\left(V\left(t_{n}^{\mathrm{sp}}\right)-V_{\text {hold }}\right) e^{-\frac{t-t_{n}^{\mathrm{sp}}}{\tau_{\mathrm{m}}}}+\frac{g_{n}^{+} \tau_{\mathrm{syn}}\left(E-V_{\mathrm{hold}}\right)}{g_{l}\left(\tau_{\mathrm{m}}-\tau_{\mathrm{syn}}\right)}\left(e^{-\frac{t-t_{n}^{\mathrm{sp}}}{\tau_{\mathrm{m}}}}-e^{-\frac{t-t_{n}^{\mathrm{sp}}}{\tau_{\mathrm{syn}}}}\right),
$$

where $t \geq t_{n}^{\mathrm{sp}}$ and $V\left(t_{0}^{\mathrm{sp}}\right)=V_{\text {hold }}$. In Eq. (13), the first to the third terms respectively corresponded to the baseline of $V$, the dynamics of $V$ in the absence of the $n$th presynaptic AP, and the effect of the $n$th presynaptic AP on $V$. Assuming that $V$ reached a peak value at $T_{n}=t_{n}^{\mathrm{sp}}+\Delta_{n}$ where $t_{n}^{\mathrm{sp}} \leq T_{n} \leq t_{n+1}^{\mathrm{sp}}$, the peak value $V\left(T_{n}\right)$ was

$$
V\left(T_{n}\right)=V_{\text {hold }}+\left(V\left(t_{n}^{\mathrm{sp}}\right)-V_{\text {hold }}\right) e^{-\frac{\Delta_{n}}{\tau_{\mathrm{m}}}}+\frac{g_{n}^{+} \tau_{\mathrm{syn}}\left(E-V_{\mathrm{hold}}\right)}{g_{l}\left(\tau_{\mathrm{m}}-\tau_{\mathrm{syn}}\right)}\left(e^{-\frac{\Delta_{n}}{\tau_{\mathrm{m}}}}-e^{-\frac{\Delta_{n}}{\tau_{\mathrm{syn}}}}\right),
$$

where

$$
\Delta_{n}=-\frac{\tau_{\mathrm{m}} \tau_{\mathrm{syn}}}{\tau_{\mathrm{m}}-\tau_{\mathrm{syn}}} \ln \left\{\frac{\tau_{\mathrm{syn}}}{\tau_{\mathrm{m}}}+\frac{g_{l}\left(\tau_{\mathrm{m}}-\tau_{\mathrm{syn}}\right)\left(V\left(t_{n}^{\mathrm{sp}}\right)-V_{\mathrm{hold}}\right)}{g_{n}^{+} \tau_{\mathrm{m}}\left(E-V_{\mathrm{hold}}\right)}\right\}
$$

The time $\Delta_{n}$ where $V(t)$ reached a peak could be obtained from the condition of $\frac{\mathrm{d} V}{\mathrm{~d} t}=0$. Finally, the synaptic response $R_{n}$ to the $n$ th-presynaptic AP was yielded as

$$
R_{n} \equiv V\left(T_{n}\right)-V\left(t_{n}^{\mathrm{sp}}\right)=\left(V_{\text {hold }}-V\left(t_{n}^{\mathrm{sp}}\right)\right)\left(1-e^{-\frac{\Delta_{n}}{\tau_{\mathrm{m}}}}\right)+\frac{g_{n}^{+} \tau_{\mathrm{syn}}\left(E-V_{\mathrm{hold}}\right)}{g_{l}\left(\tau_{\mathrm{m}}-\tau_{\mathrm{syn}}\right)}\left(e^{-\frac{\Delta_{n}}{\tau_{\mathrm{m}}}}-e^{-\frac{\Delta_{n}}{\tau_{\mathrm{syn}}}}\right) .
$$

When a sequence of presynaptic APs was regular, the synaptic response $R_{n}$ fell into a steady-stage value for $n \rightarrow \infty$. From Eq. (16), the steady-state value $R_{\infty}$ could be described as

$$
R_{\infty}=\left(V_{\text {hold }}-V\left(t_{\infty}^{\mathrm{sp}}\right)\right)\left(1-e^{-\frac{\Delta \infty}{\tau_{\mathrm{m}}}}\right)+\frac{g_{\infty}^{+} \tau_{\mathrm{syn}}\left(E-V_{\mathrm{hold}}\right)}{g_{l}\left(\tau_{\mathrm{m}}-\tau_{\mathrm{syn}}\right)}\left(e^{-\frac{\Delta_{\infty}}{\tau_{\mathrm{m}}}}-e^{-\frac{\Delta_{\infty}}{\tau_{\mathrm{syn}}}}\right),
$$

where 


$$
\Delta_{\infty}=-\frac{\tau_{\mathrm{m}} \tau_{\mathrm{syn}}}{\tau_{\mathrm{m}}-\tau_{\mathrm{syn}}} \ln \left\{\frac{\tau_{\mathrm{syn}}}{\tau_{\mathrm{m}}}+\frac{g_{l}\left(\tau_{\mathrm{m}}-\tau_{\mathrm{syn}}\right)\left(V\left(t_{\infty}^{\mathrm{sp}}\right)-V_{\mathrm{hold}}\right)}{g_{\infty}^{+} \tau_{\mathrm{m}}\left(E-V_{\mathrm{hold}}\right)}\right\}
$$

The values of $g_{\infty}^{+}$and $V\left(t_{\infty}^{\mathrm{sp}}\right)$ in Eqs. (17) and (18) were the steady-state values of $g_{n}^{+}$and $V\left(t_{n}^{\mathrm{sp}}\right)$ and could be obtained from Eqs. (12) and (13) because $g_{\infty}^{+}=g_{n}^{+}=g_{n+1}^{+}$and $V\left(t_{\infty}^{\mathrm{sp}}\right)=V\left(t_{n}^{\mathrm{sp}}\right)=V\left(t_{n+1}^{\mathrm{sp}}\right)$ were satisfied under the steady state. Besides, the situation of a regular presynaptic AP train allowed its inter-spike intervals (ISI) to be constant, so that $\Delta t_{n}=\Delta t$. Then,

$$
\begin{aligned}
g_{\infty}^{+} & =\frac{\bar{g} u_{\infty}^{+} x_{\infty}^{-}}{1-e^{-\frac{\Delta t}{\tau_{\mathrm{syn}}}}}, \\
V\left(t_{\infty}^{\mathrm{sp}}\right) & =V_{\text {hold }}+\frac{g_{\infty}^{+} \tau_{\text {syn }}\left(E-V_{\mathrm{hold}}\right)}{g_{l}\left(\tau_{\mathrm{m}}-\tau_{\mathrm{syn}}\right)} \cdot \frac{e^{-\frac{\Delta t}{\tau_{\mathrm{m}}}}-e^{-\frac{\Delta t}{\tau_{\mathrm{syn}}}}}{1-e^{-\frac{\Delta t}{\tau_{\mathrm{m}}}}} .
\end{aligned}
$$

In the same manner with $g_{\infty}^{+}$and $V\left(t_{\infty}^{\mathrm{sp}}\right)$, the steady-state values $u_{\infty}^{+}$and $x_{\infty}^{-}$were yielded from Eqs. (9) and (10), and were as followings:

$$
\begin{aligned}
& u_{\infty}^{+}=\frac{1}{2 \epsilon}\left(\sqrt{\zeta^{2}-4 \epsilon \eta}-\zeta\right), \\
& x_{\infty}^{-}=\frac{e^{\frac{\Delta t}{\tau_{d}}}-1}{e^{\frac{\Delta t}{\tau_{d}}}+u_{\infty}^{+}-1},
\end{aligned}
$$

where $\epsilon=\alpha\left\{1+(a \sqrt{U}-1) e^{-\frac{\Delta t}{\tau_{f}}}\right\} e^{-\frac{\Delta t}{\tau_{f}}}, \zeta=b+\{\sqrt{U}(a b-\alpha a-1)-b\} e^{-\frac{\Delta t}{\tau_{f}}}$, and $\eta=\sqrt{U}(1-a b)$. In Eqs. (19)-(22), using the mean firing rate $\lambda$, the ISI $\Delta t$ was represented as $\Delta t=10^{3} / \lambda$ since the unit of $\Delta t$ was milliseconds.

\subsection{Map function analysis of discretized STP model}

In the previous section, the augmented STP model defined as Eqs. (1) and (5) was transformed to the iterative expressions described in Eqs. (9) and (10). The iterative expressions permitted me to analyze the augmented STP model with a map function. A shape of a map function of $u$ was one of the important factors to determine synaptic responses. To reveal the mechanisms of the FG and the $\mathrm{FD}$, the map function of the variable $u^{+}$was analyzed since the $u^{+}$was dominant in the two cases. Here, let me define the map function of $u$ as $h(u)$ from Eq. (10). Then, the $h(u)$ could be written as

$$
h(u)=u e^{-\frac{\Delta t}{\tau_{f}}}+\sqrt{U}\left(1-u e^{-\frac{\Delta t}{\tau_{f}}}\right)\left(a-\frac{1}{\alpha u e^{-\frac{1}{\lambda \tau_{f}}}+b}\right),
$$

then, Eq. (10) enabled to be written as $u_{n+1}^{+}=h\left(u_{n}^{+}\right)$.

The slope of the map function described by Eq. (23) at $u=0$ was one of the statistics to characterize synaptic responses for regular presynaptic AP trains. From Eq. (23), the slope of the map function was yielded as

$$
\left.\frac{\mathrm{d} h}{\mathrm{~d} u}\right|_{u=0}=e^{-\frac{\Delta t}{\tau_{f}}}\left(1-U+\frac{\alpha \sqrt{U}}{b^{2}}\right) .
$$

\subsection{Identification of FG and FD}

To characterize the dependence of the FG and the FD on a parameter space of $U$ and $\alpha$ in the augmented STP model, the FG and the FD behaviors should be identified. The identification of these behaviors came from eight synaptic responses to a regular presynaptic AP train. All the synaptic responses for the identification were normalized by the first response.

The FG is a phenomenon where synaptic responses to a few APs in a regular AP train continue to grow supuralinearly (See also Sec. 3.1 and Refs. [23,26]). To identify this property in synaptic responses, the synaptic responses were fit by a quadratic function of $p_{1} n^{2}+p_{2} n+p_{3}$. On the fitting, the nonlinear least square method was employed. When synaptic responses grew supralinearly in the 
FG, the fitting parameter $p_{1}$ would be positive if the function was well fit to the data of the synaptic responses.

Compared with the FG, the FD behavior was more complicated. When synaptic responses to a regular AP train exhibited the FD, early (typically the second and the third) responses were strongly facilitated and their following responses were depressed compared with the facilitated responses (See also Sec. 3.1 and Ref. [16]). Consequently, the seventh and the eighth responses got as small as the first response or less. To quantify such a complicated synaptic behavior, two statistical tests were conducted. First, a one-sample and one-sided t-test identified a part of the facilitation. In this first statistical test, the first response, the largest response, and the second-largest response were eliminated from all the synaptic responses and these data were regarded as a sample. An alternative hypothesis of the first test $\left(H_{1}^{(1)}\right)$ was that the mean value of the sample was significantly smaller than the mean value of the largest and the second-largest response. Next, one-sample and two-sided t-test was also conducted. In this second test, the sample was the same data with the first test but the mean value of the sample was compared with the first response. Then, an alternative hypothesis of the second test $\left(H_{1}^{(2)}\right)$ was the significant difference in the mean value of the sample from the first response. When the alternative hypothesis was accepted in the second test, whether the mean value was significantly smaller or larger than the first response was decided by comparing the first response. By combining the results of the two statistical tests, four modes of synaptic responses were distinguishable. Observed synaptic responses and their corresponding modes were summarized in Table I. In the current study, modes 1 and 3 were regarded as the FD because synaptic responses were strongly facilitated and then strongly depressed (depressed responses are same as the first response (mode 1) and smaller then the first response (mode 3)). It was confirmed the largest response were always the second or the third one in the range of the parameters tested in the current study (Data not shown).

Table I. Relation between mode of STP model in FD synapse and results of the statistical test. The checkmarks indicates the alternative hypothesis is accepted and otherwise the blanks. The dashes indicates not-evaluated. $\tilde{R}$ is the mean value of the sample data estimated in the second statistical test.

\begin{tabular}{|c|c|c|c|c|}
\hline mode & 0 & 1 & 2 & 3 \\
\hline \hline$H_{1}^{(1)}$ & & $\checkmark$ & $\checkmark$ & $\checkmark$ \\
$H_{1}^{(2)}$ & - & & $\checkmark$ & $\checkmark$ \\
$\tilde{R}<R_{1}$ & - & - & & $\checkmark$ \\
\hline
\end{tabular}

\subsection{Tuning curves}

To characterize synaptic responses to random presynaptic AP trains, tuning curves were introduced. A tuning curve was a function of a postsynaptic response against an ISI between a presynaptic AP evoking the response and it previous AP. In other words, the tuning curve was the relation between $R_{n}$ and $\Delta t_{n-1}=t_{n}^{\mathrm{sp}}-t_{n-1}^{\mathrm{sp}}$. However, the evoked responses fluctuated due to the difference in the internal states of the system, even if presented ISIs were identical. Then, the tuning curve was created in the following manner:

1. All ISIs observed from a simulation were categorized into 20 groups, which categorized similar ISIs into the same group.

2. By referring to the corresponding or the paired ISIs, postsynaptic responses were categorized into 20 groups. Then, the responses triggered by similar ISIs were in the same group.

3. The postsynaptic responses in the same group were merged and then averaged over.

The bin width was $40 \mathrm{~ms}$ for $\lambda=10 \mathrm{sp} / \mathrm{s}$ presynaptic AP trains and was $10 \mathrm{~ms}$ for $\lambda=40 \mathrm{sp} / \mathrm{s}$ presynaptic AP trains to obtain the mean response of the categorized ISIs. 


\subsection{Information theoretical analysis}

As a simple way to characterize synaptic responses to random presynaptic AP trains, the tuning curve was utilized in the previous section. To more deeply understand the relation between $\Delta t_{n-1}$ and $R_{n}$, the information-theoretic analysis was conducted. For the analysis, mutual information (MI) was employed. The MI of $I(X ; Y)$ was defined as

$$
I(X ; Y)=\int_{y \in Y} \int_{x \in X} p(x, y) \log _{2} \frac{p(x, y)}{p(x) p(y)} \mathrm{d} x \mathrm{~d} y,
$$

where $p(x)$ and $p(y)$ were the marginal probability density functions (PDFs) of $X$ and $Y$ and $p(x, y)$ was the joint PDF of $X$ and $Y$. In the present study, $X$ was a set of $R_{n}$ and $Y$ was a vector of $\Delta t_{n-1}$. Bin widths to estimate PDFs were $1 \%$ of the maximum values of $X$ and $Y$.

\section{Results}

\subsection{Synaptic responses against regular presynaptic AP trains}

As the first step of analyses, four types of synaptic responses of the augmented STP model to regular AP trains are observed: depression, facilitation, facilitation growth (FG), and facilitation-depression (FD) (Figs. 2-5). For the convenience of comparing with the empirical results in Refs. [16, 23, 26], regular AP trains with 10 and $40 \mathrm{sp} / \mathrm{s}$ are applied to the four synapses.

The synaptic response of the model is slightly depressed for the low presynaptic AP rate when $U$ is large and $\tau_{d}>\tau_{f}$ (top panel of Fig. $2(\mathrm{~A})$ ). Because the time constant of $x$ is twice as the rate of the presynaptic APs, the next presynaptic AP comes before the convergence to the default value of $x$ (bottom panel of Fig. 2(A)). The variable $x^{-}$then gradually decreases at every presynaptic AP arrivals. In contrast, the time constant of $u$ is four times smaller than the presynaptic AP rate, so that the variable $u$ almost goes back to the baseline after presynaptic APs (middle panel of Fig. 2(A)). That is why the variable $u^{+}$keeps almost constant value at presynaptic AP arrivals. The response more reflects the values of $x^{-}$in the synapse. For the higher presynaptic AP rate, the synapse exhibits the strong depression (top panel of Fig. 2(B)). In this case, the time constant of $x$ is eight times larger than the presynaptic AP rate. Therefore, $x^{-}$converges to a smaller value than that for the lower presynaptic AP rate (bottom panel of Fig. 2(B)). The variable $u$ has the same time constant with the presynaptic AP rate, and then the convergence of $u^{+}$converges to almost the same value with that of the lower presynaptic AP rate (middle panel of Fig. 2(B)). In both cases, the synapse displays simple behaviors. The presynaptic efficacy monotonically decreases and converges to a steady-state value until the eighth presynaptic AP for the repetitive presynaptic APs (Fig. 2(C)). The convergent value of the synaptic responses for the higher presynaptic AP rate is about three times as small as that for the lower presynaptic AP rate.

The model synapse displays facilitation when $U$ is small and $\tau_{f}>\tau_{d}$ (top panels of Fig. 3(A), (B)). Different from the depression synapse, the postsynaptic responses reflect the kinetics of $u$ rather than that of $x$ (middle and bottom panels of Fig. 3(A), (B)). The higher presynaptic AP rate leads the synapse to stronger facilitation (Fig. $3(\mathrm{C})$ ).

Typical responses of synaptic depression and facilitation have already been shown and the results demonstrated in the previous sections can also be generated in the canonical model. The canonical model, however, cannot reproduce some STP phenomena observed in electrophysiological studies. One of the phenomena is the FG. In typical facilitation synapses, $R_{n} / R_{1}$ against the presynaptic AP number shows saturation as shown in Fig. 3(C). Contrary, a later presynaptic AP evokes a larger postsynaptic response in the FG at the early state of presynaptic AP stimuli. The FG is observable in excitatory synapses on low-threshold spiking neurons in layer 4 rat somatosensory cortex [23] and the most proximal muscle fibers in crayfish [26]. The linearity in the canonical model might prevent the realization of such synaptic behaviors. The nonlinearity has been introduced to the update function of $u$ triggered by presynaptic APs of TM model as in Eq. (5).

The augmented model succeeds to generate the FG when $U$ is very small and $\tau_{f} \gg \tau_{d}$ (top panel in Fig. 4(B)). In both kinetics of $x$ and $u$, the later presynaptic AP induces the larger degree of the update (middle and bottom panels of Fig. 4(B)) and the amplitude of the postsynaptic responses 
A
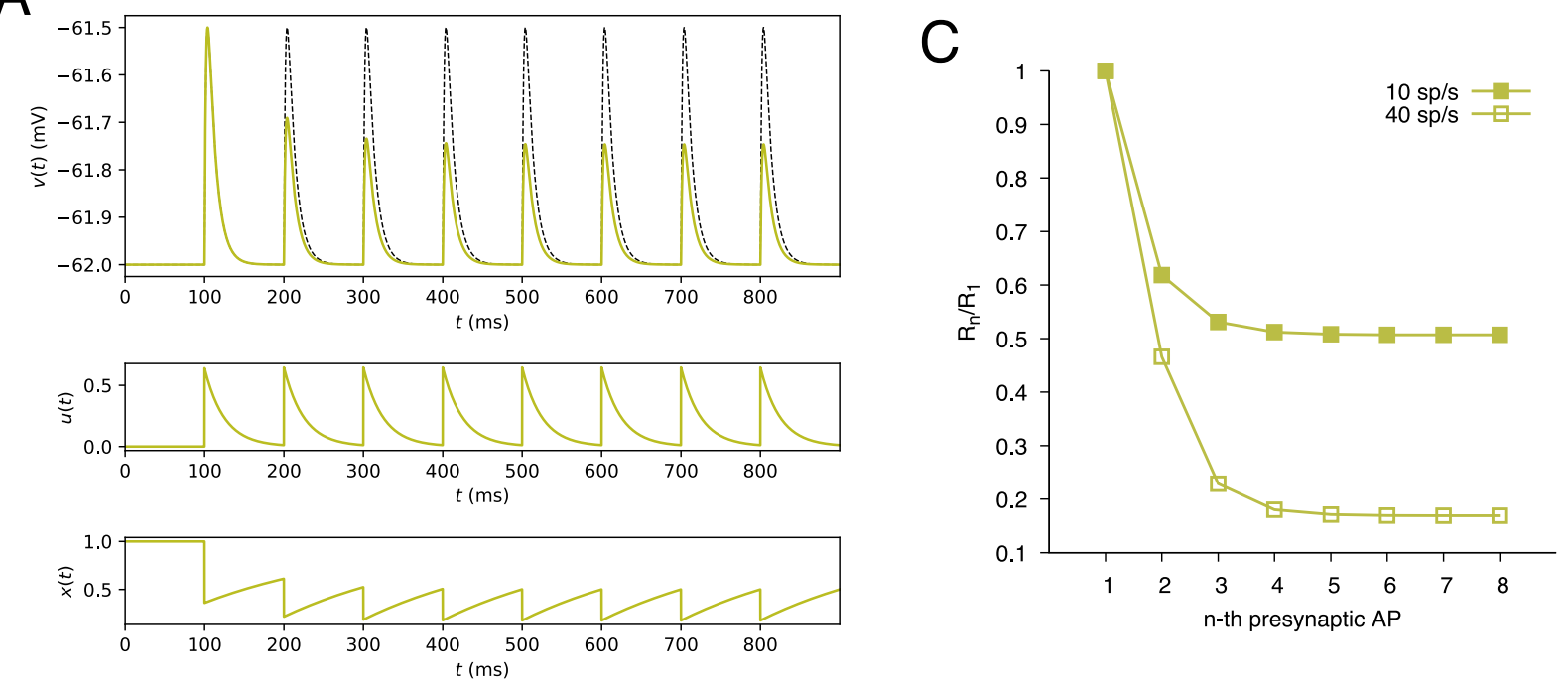

B
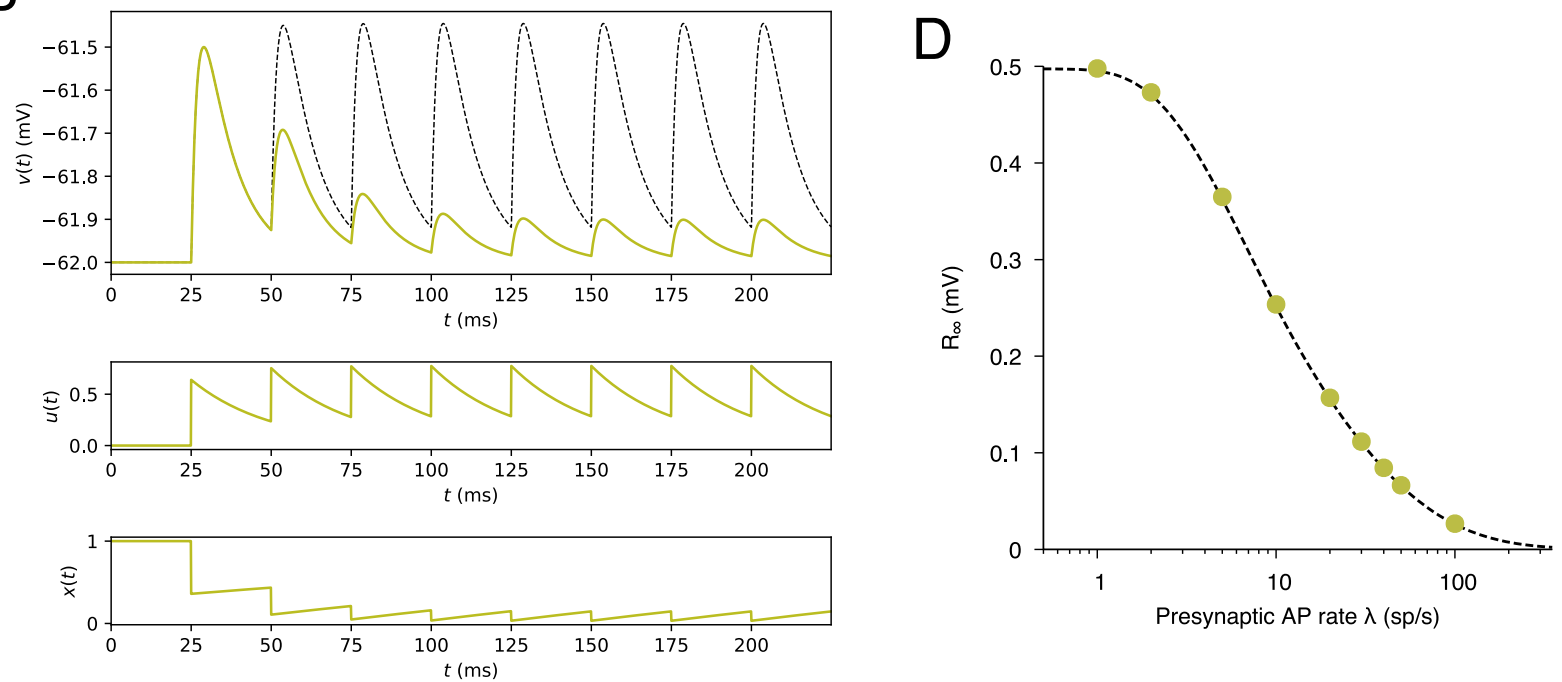

Fig. 2. Typical responses of depression synapses to regular presynaptic AP trains. Presynaptic AP rate is (A) $10 \mathrm{sp} / \mathrm{s}$ and (B) $40 \mathrm{sp} / \mathrm{s}$. In the top panels in (A) and (B), the responses of a synapse without STP (black-dashed lines) are plotted as the guideline. (C) A function of the normalized response amplitude against the presynaptic AP number. (D) The steady state value of the response amplitude in the depression synapse. The circles are the values obtained from a simulation and the dashed line is the theoretical estimation of Eq. (17). The parameters of all the models are set as $\bar{g}=1.88 \mathrm{nS}, U=0.64, \tau_{d}=200 \mathrm{~ms}$, $\tau_{f}=25 \mathrm{~ms}$, and $\alpha=0.001$.

continues to supralinearly grow until the eighth presynaptic AP (Fig. 4(C)). The response evoked by the eighth presynaptic AP is much larger compared with the normal facilitation synapse (Figs. 3 and $4(\mathrm{C}))$. Contrary to the high presynaptic AP rate, the synaptic responses for the low presynaptic AP rate is quite similar to the responses of the facilitation synapse and both synapses exhibit sublinear growth of responses (Figs. 3 and 4(A)). FG is then only observable for a relatively high presynaptic AP rate (Fig. 4(A)-(C)).

The FD, that is not reproducible in the canonical model, is another phenomenon and is observed in Schaffer collateral to CA1 pyramidal synapses. In the FD, the second or the third presynaptic AP induces the strong facilitation of postsynaptic responses and the responses to the latter presynaptic APs are strongly depressed [16]. Analogous to the FG phenomenon the FD phenomenon can be reproduced in the augmented model in the case of high presynaptic AP rate (Fig. 5(A)-(C)). For the low presynaptic AP rate, the second and the third synaptic responses are the largest and the 

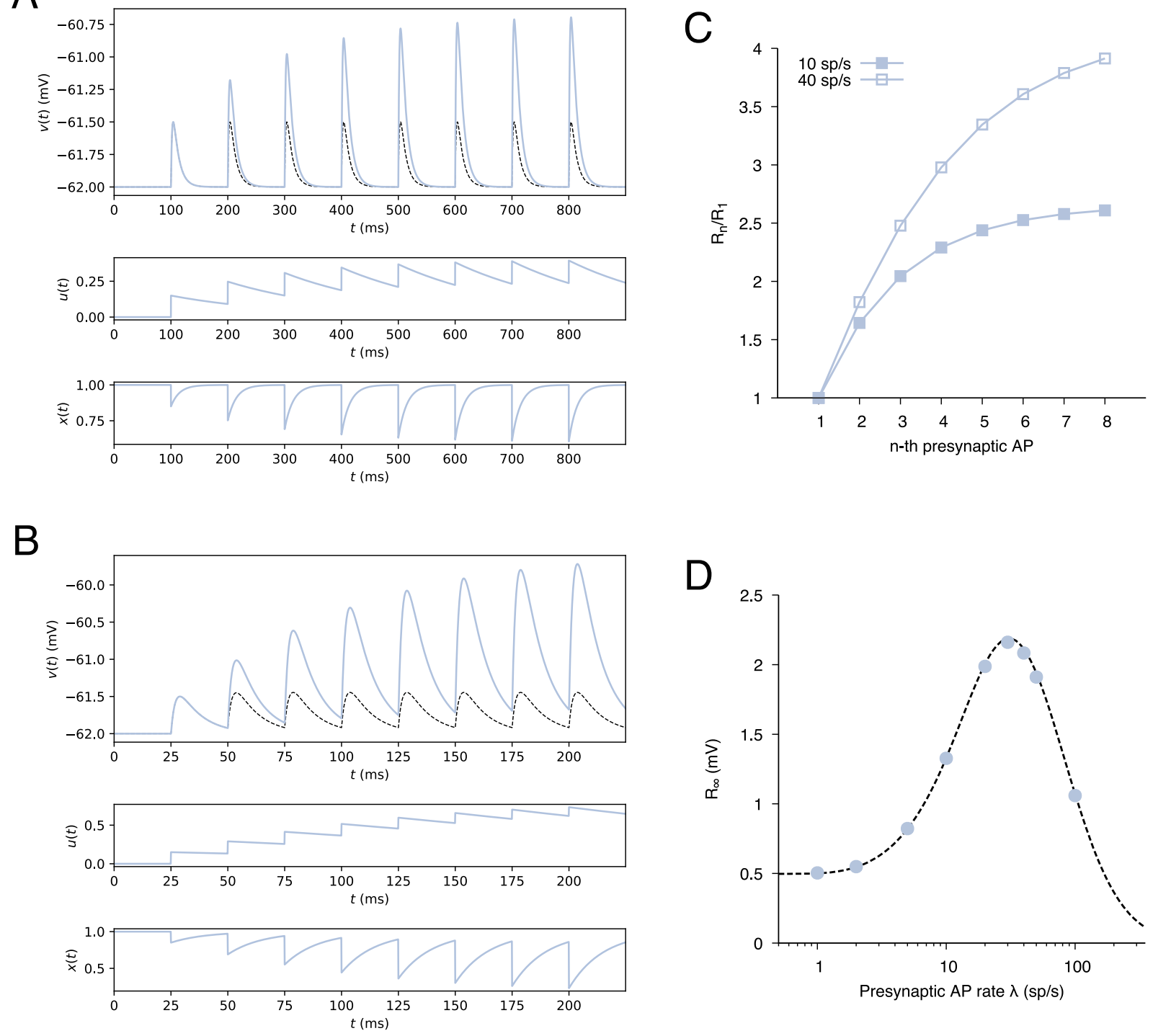

Fig. 3. Typical behaviors of facilitation synapses to regular presynaptic AP trains. Presynaptic AP rate is (A) $10 \mathrm{sp} / \mathrm{s}$ and (B) $40 \mathrm{sp} / \mathrm{s}$. In the top panels in (A) and (B), the responses of a synapse without STP (black-dashed lines) are plotted as the guideline. (C) A function of the normalized response amplitude against the presynaptic AP number. (D) The steady state value of the response amplitude in the facilitation synapse. The circles are the values obtained from a simulation and the dashed line is the theoretical values of Eq. (17). The parameters of all the models are set as $\bar{g}=8 \mathrm{nS}, U=0.15, \tau_{d}=15 \mathrm{~ms}$, $\tau_{f}=200 \mathrm{~ms}$, and $\alpha=0.001$.

latter responses are slightly depressed. The following responses gradually get smaller than the third response but are much larger than the first response. For the higher presynaptic AP rate, the synaptic responses are qualitatively similar for the lower presynaptic AP rate. The responses after the third response are, however, strongly depressed and are as same as the first response.

\subsubsection{Steady-state analysis in postsynaptic responses for regular presynaptic AP trains}

As a next step of the characterization of the synapses, it is analyzed the steady-state value $R_{\infty}$ of synaptic responses to regular presynaptic AP trains in different frequencies (Figs. 2-5(D)). Figures 25 (D) display theoretical and numerical predictions of $R_{\infty}$ for the presynaptic AP rate ranging between 0.5 and $350 \mathrm{sp} / \mathrm{s}$.

In the case of the depression synapse, $R_{\infty}$ does not change from the first response $(0.5 \mathrm{mV})$ for less than the presynaptic AP rate of $2 \mathrm{sp} / \mathrm{s}$ in the depression synapse (Fig. 2(D)). For the higher 
A
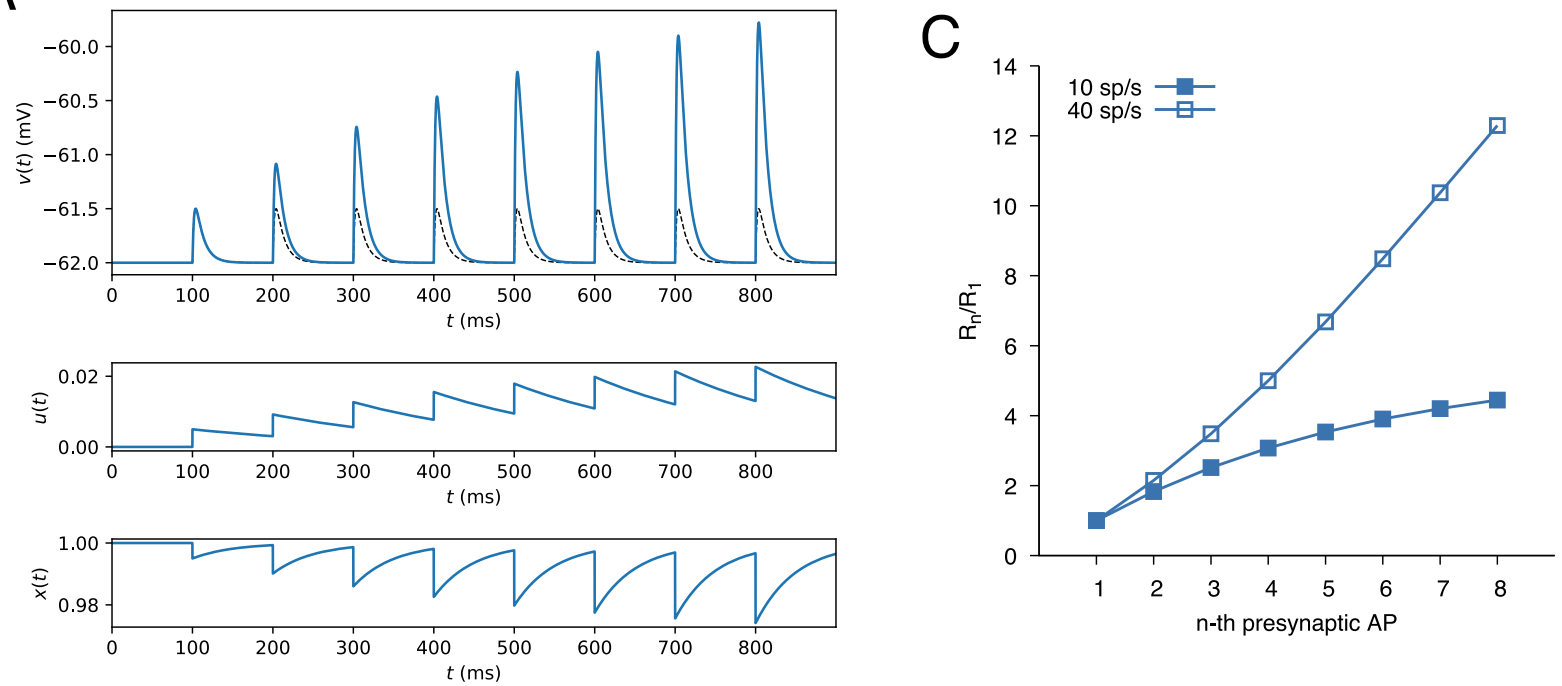

B
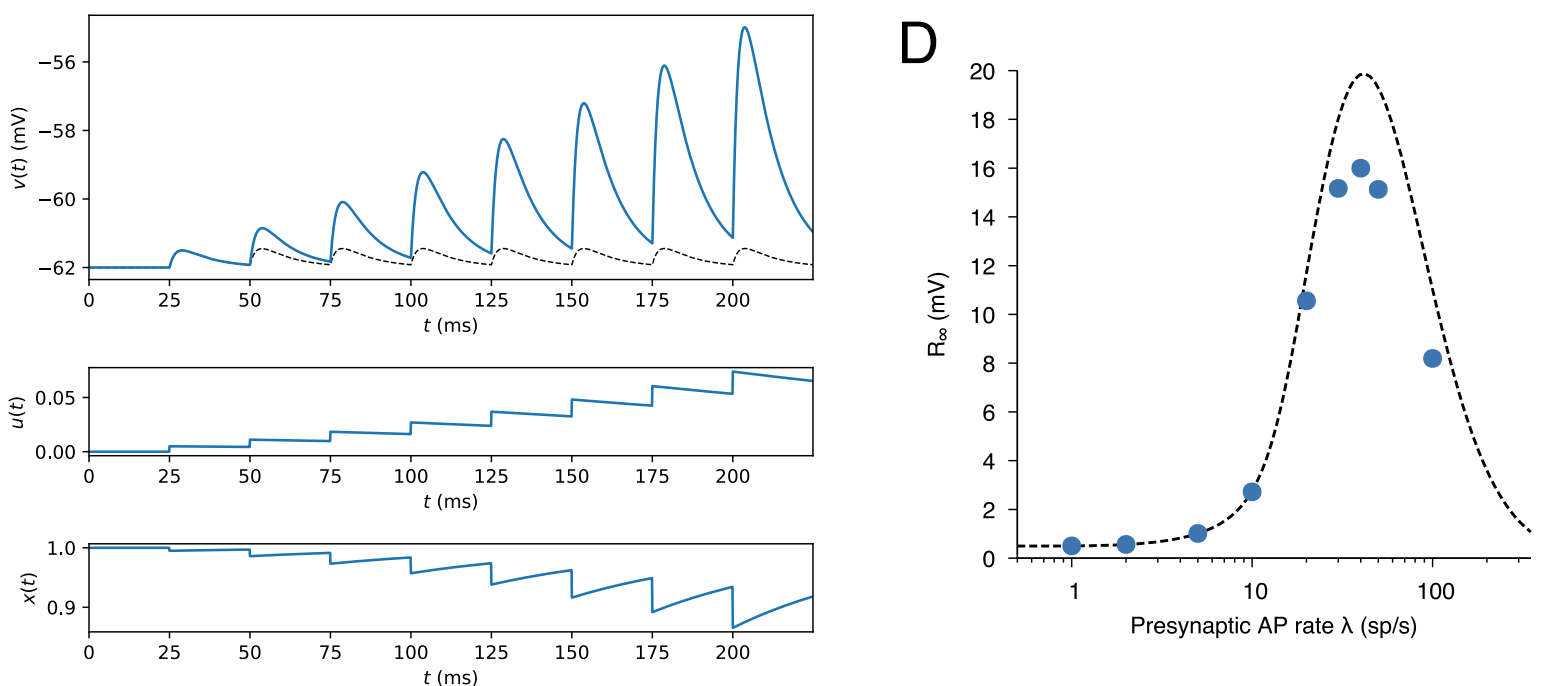

Fig. 4. Examples of facilitation growth to regular presynaptic AP trains. Presynaptic AP rate is (A) $10 \mathrm{sp} / \mathrm{s}$ and (B) $40 \mathrm{sp} / \mathrm{s}$. In the top panels in (A) and (B), the responses of a synapse without STP (black-dashed lines) are plotted as the guideline. (C) A function of the normalized response amplitude against the presynaptic AP number. (D) The steady state value of the response amplitude in the synapse exhibiting the facilitation growth. The circles are the values obtained from a simulation and the dashed line is the theoretical values of Eq. (17). The parameters of all the models are set as $\bar{g}=0.24 \mu \mathrm{S}, U=0.005$, $\tau_{d}=50 \mathrm{~ms}, \tau_{f}=200 \mathrm{~ms}$, and $\alpha=4.5$.

presynaptic AP rate, $R_{\infty}$ monotonically decreases. In contrast, there is a peak in the function of $R_{\infty}$ against the presynaptic AP rate in the other types of the facilitation synapses (Figs. 3-5(D)). This indicates that an optimal presynaptic AP rate exists for facilitation synapses.

In all the cases except for the FG synapse, the theoretical values of $R_{\infty}$ successfully predict and trace the numerically obtained values (Figs. 2-5(D)). In the FG case, the theory fails to predict $R_{\infty}$ obtained from numerical simulations around the peak of the function (Fig. 4(D)). The reason why the theory fails to predict $R_{\infty}$ for the high presynaptic AP rate is the assumption in yielding the theoretical predictions of the steady-state value. To yield the theoretical values, $g(E-V)$ is approximated as $g\left(E-V_{\text {hold }}\right)$ in Eq. (13). However, this assumption is only satisfied under the condition where the postsynaptic potential is close to $V_{\text {hold }}$. Around the peak of the function, the next presynaptic AP arrives before $V$ goes back to $V_{\text {hold }}$ enough because the presynaptic AP effect is extremely large. Under this situation, the assumption of the approximation is no longer satisfied. 
A
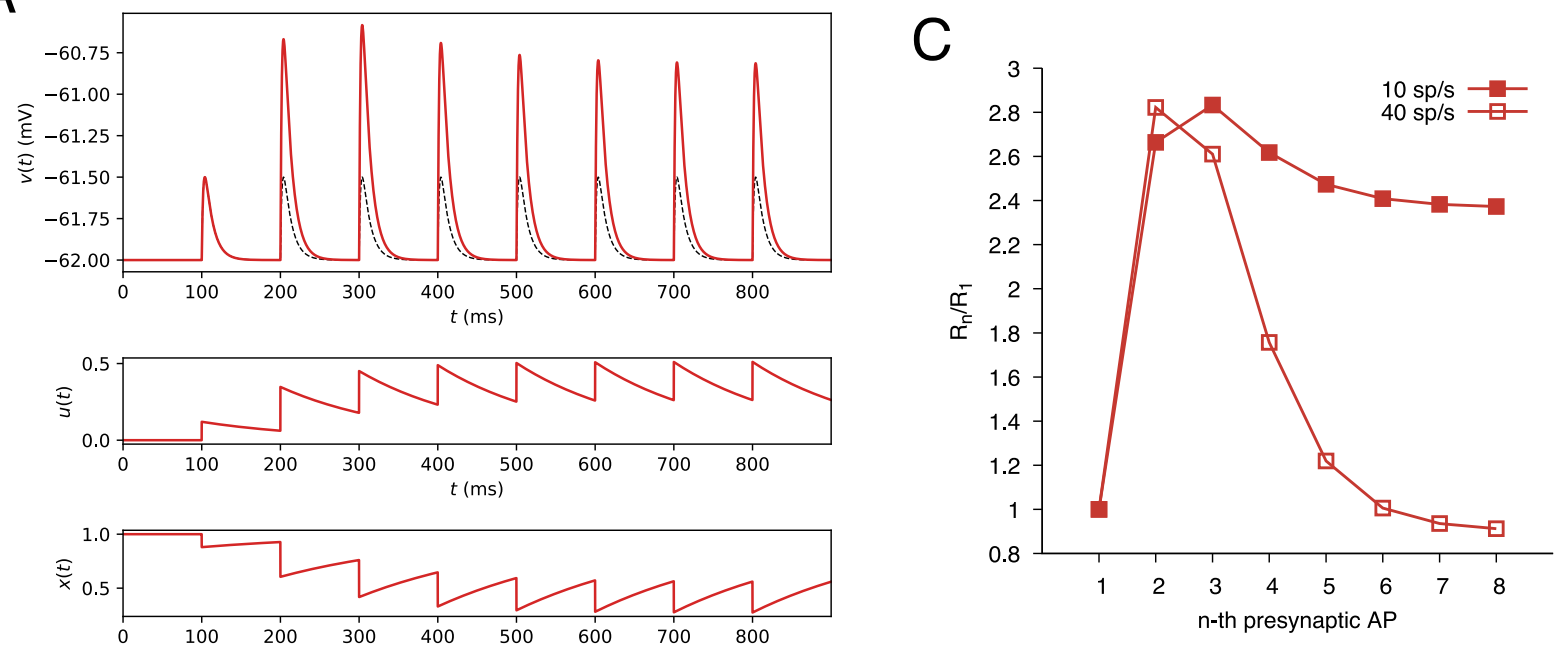

B
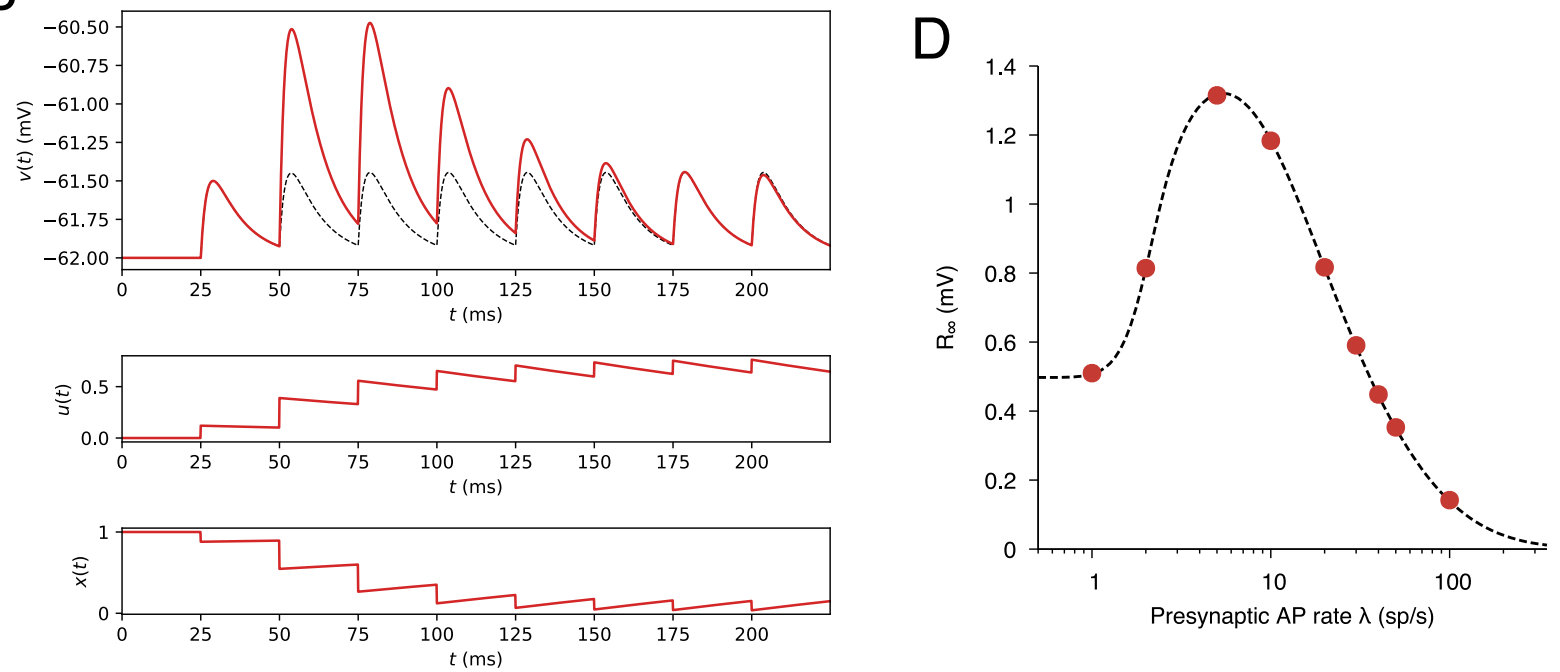

Fig. 5. Examples of facilitation-depression to regular presynaptic AP trains. Presynaptic AP rate is (A) $10 \mathrm{sp} / \mathrm{s}$ and (B) $40 \mathrm{sp} / \mathrm{s}$. In the top panels in (A) and (B), the responses of a synapse without STP (black-dashed lines) are plotted as the guideline. (C) A function of the normalized response amplitude against the presynaptic AP number. (D) The steady state value of the response amplitude in the synapse exhibiting the facilitation and the depression. The circles are the values obtained from a simulation and the dashed line is the theoretical values of Eq. (17). The parameters of all the models are set as $\bar{g}=10 \mathrm{nS}, U=0.12, \tau_{d}=200 \mathrm{~ms}, \tau_{f}=150 \mathrm{~ms}$, and $\alpha=100$.

\subsubsection{Mechanisms of FG and FD}

In the previous section, it has already been shown the augmented STP model can reproduce both FG and FD (Figs. 4 and 5). The AP effect in the variable $u$ of the augmented STP model has the nonlinearity (Fig. 1(B)), however, it is still unclear how the FG and the FD are realized. To understand the mechanisms of the FG and the FD, not only the AP effect in $u$ but also the dynamics of whole $u$, especially $u^{+}$, should be analyzed. Since the differential equation of the variable $u$ in Eq. (5) has been transformed into the iterative expression of Eq. (10), the map function analysis is applicable. The map function of Eq. (10) can be described as Eq. (23).

The map functions of all the three types monotonically increase as $u_{n}^{+}$increases (Fig. 6). While the monotonic increase of the map function is a common property among all the types, an alternative property is observed only in the cases of the FG synapse with a high presynaptic AP rate (Fig. 4 lower panel) and the FD synapse (Fig. 5). In these cases, the map function has the slope at $u=0$ 
A

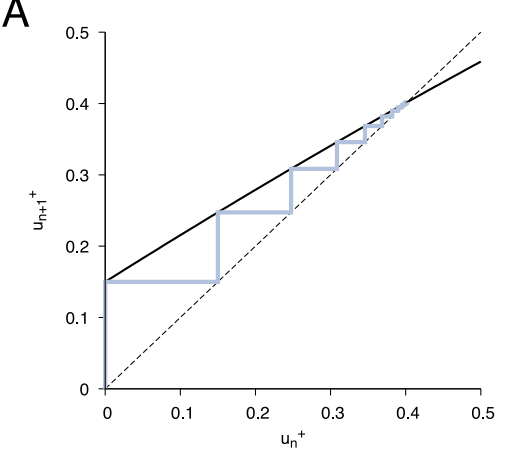

B
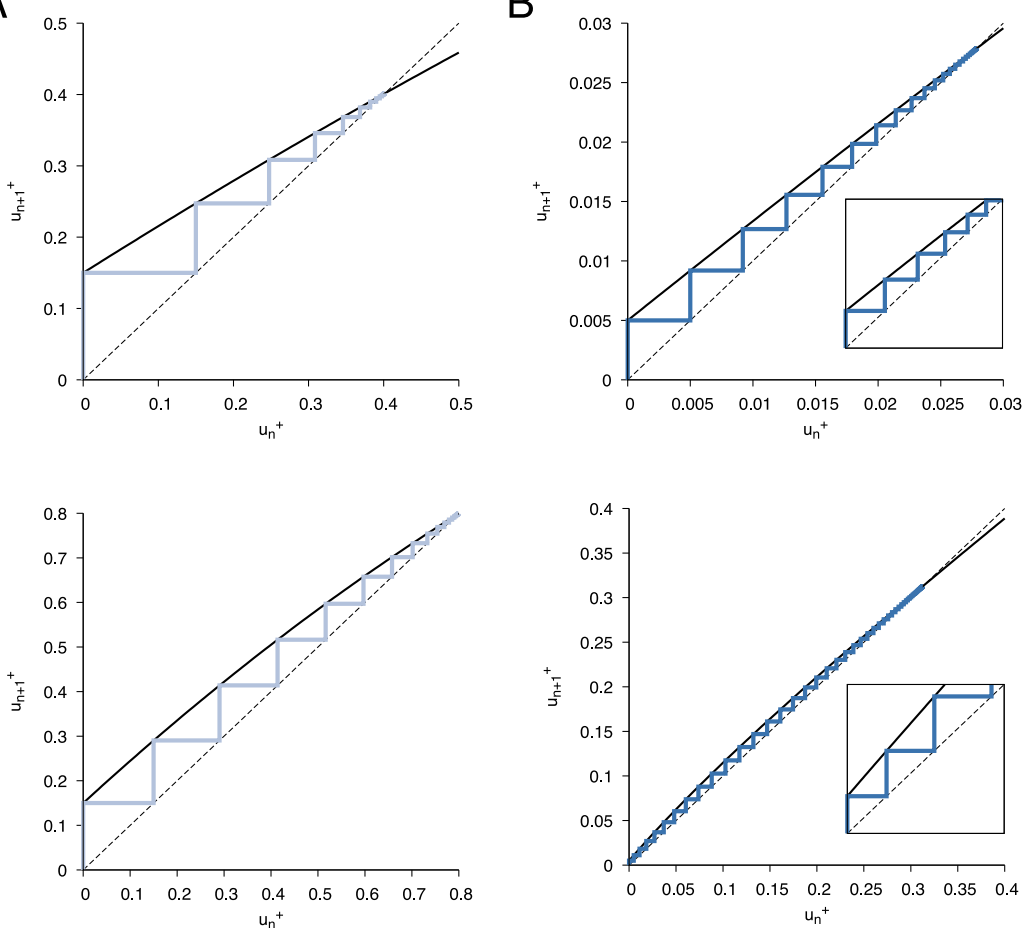

C
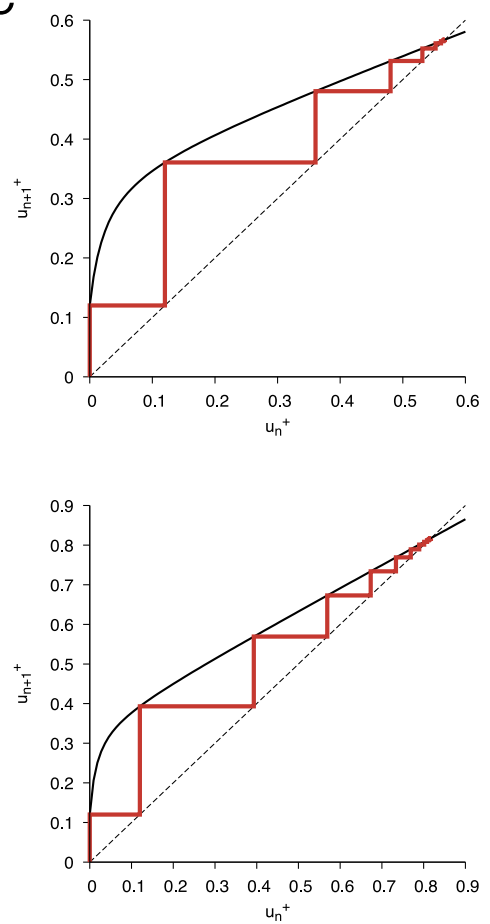

Fig. 6. Map function $h\left(u_{n}^{+}\right)$(black solid lines) and trajectory of $u_{n}^{+}$(colored lines) of (A) facilitation synapse, (B) FG synapse, and (C) FD synapse. The parameters of these synapses are the same as Figs. 3-5. The upper and the lower panels are maps in cases of 10 and $40 \mathrm{sp} / \mathrm{s}$ rate of presynaptic APs. A black dashed line represents $u_{n}^{+}=u_{n+1}^{+}$. In (B), the insets are the enlargements in the area of $0 \leq u_{n}^{+} \leq 0.02$ and $0 \leq u_{n+1}^{+} \leq 0.02$.

A

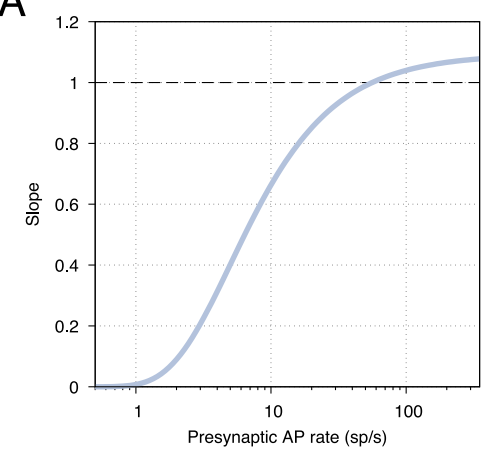

B

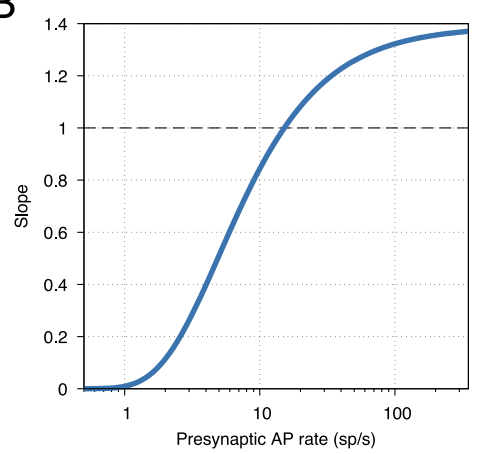

C

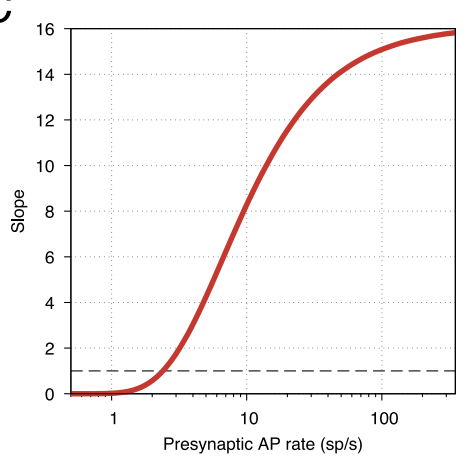

Fig. 7. The dependency of the slope of the map function at $u=0$ on the presynaptic AP rate described in Eq. (24). (A) facilitation synapse, (B) FG synapse, and (C) FD synapse. The parameters of these synapses are the same as Figs. 3-5. In each panel, the dashed lines are the baseline of unity.

larger than unity. Contrary, the slope is less than unity in the other cases (Fig. 6(B) upper panel and (C)). Besides, the slope is not only determined by the parameter values of the STP model but also is influenced by the presynaptic AP rate (Fig. 4). The theoretically yielded value of the slope of $h(u)$ at $u=0$ includes a factor of the presynaptic AP rate in Eq. (24). The function of the slope against the presynaptic AP rate is plotted to check how the slope changes with the presynaptic AP rate.

In the case of the facilitation synapse, the slope takes over one at $60 \mathrm{sp} / \mathrm{s}$ of the presynaptic AP rate (Fig. 7A). Even though the presynaptic AP rate is higher, the slope does not get larger and almost converges to 1.1. Then, this type of synapse does not exhibit supralinear growth of responses such as the FG and the FD property to the presynaptic AP trains with 10 and $40 \mathrm{sp} / \mathrm{s}$ (Fig. 3(A), (B)).

The presynaptic AP train with $15 \mathrm{sp} / \mathrm{s}$ allows the slope of the FG synapse to be larger than one (Fig. 7B). This accounts for the sublinear growth of responses to $10 \mathrm{sp} / \mathrm{s}$ presynaptic AP trains and 
the supralinear growth of responses to $40 \mathrm{sp} / \mathrm{s}$ presynaptic AP trains (Fig. 4(A), (B)). Additionally, the slope becomes more than 1.2 when the presynaptic AP rate is over $30 \mathrm{sp} / \mathrm{s}$, different from the case of the facilitation synapse. This is the reason why the supralinear growth of responses is only observed for the high presynaptic AP rate.

The presynaptic AP rate of $3 \mathrm{sp} / \mathrm{s}$ is only needed for the slope taking over one in the FD synapse (Fig. 7C). Therefore, the FD synapse exhibits the prominent facilitation in the second response for both 10 and $40 \mathrm{sp} / \mathrm{s}$ presynaptic AP trains (Fig. 5(A), (B)). The second response is prominently facilitated but the facilitation in the third response is slightly weakened. This is because the variable $u_{n}^{+}$passes through the close point where $h\left(u_{n}^{+}\right)-u_{n}^{+}$is the largest. In addition to this, the dominance of the variables $x$ and $u$ switches resulting from the different time scales of these variables. The facilitation effect is, then, weaker in the synaptic responses to later presynaptic APs and the synaptic response is consequently depressed. This is the facilitation-depression switching mechanism in the FD synapse.

\subsubsection{Parameter dependency of FG and FD}

In the previous section, the mechanisms of the FG and the FD have been analyzed and it has been shown that the shape of the map function $h(u)$ defined in Eq. (23), especially the slope of $h(u)$ at $u=0$ described in Eq. (24) is important to decide synaptic responses in the augmented STP model. The synaptic responses realized by the STP model, however, might drastically be dependent on its parameter values. In this section, it is numerically investigated how the synaptic responses of the FG and the FD vary on the parameter space of $U$ and $\alpha$. Besides, how the variation of the synaptic responses are affected by the presynaptic AP rate is also evaluated.

For $10 \mathrm{sp} / \mathrm{s}$, the FG phenomenon never occurs in the parameter space. Conversely, when $40 \mathrm{sp} / \mathrm{s}$, the STP model exhibits stronger FG phenomena for smaller $U$ and larger $\alpha$ (Fig. 8(A), (B)). More precisely, the values of $U$ larger than 0.015 does not lead the FG to the STP model. Additionally, even though $U<0.015$ is satisfied, $\alpha<1.5$ induces no FG, indicating that the nonlinearity in the STP model plays a crucial role for the realization of the FG.

When the presynaptic AP arrivals are low rate $(10 \mathrm{sp} / \mathrm{s})$, the STP model does not show the FD phenomena in the wide range of the parameter space (Fig. 8(C)). The desired phenomena appear for $U>0.33$. A great influence of $\alpha$ on the synaptic responses is observed for $\alpha<30$ and a little influence is apparent for the larger $\alpha$. When presynaptic APs arrive in shorter time $(40 \mathrm{sp} / \mathrm{s})$, the FD region is made larger (Fig. 8(D)). Besides, the influence of the change of $\alpha$ decreases.

\subsection{Synaptic responses against random presynaptic AP trains}

In the previous sections, the synaptic responses to regular presynaptic AP trains have been demonstrated and characterized in respects with instantaneous and a steady-state behaviors. In more realistic situations, however, presynaptic neuronal activities are not regular, and random presynaptic AP trains seem to be more appropriate for the conditions [33]. In the present section, synaptic responses of the augmented STP model to random presynaptic AP trains are evaluated (Fig. 9).

\subsubsection{Mean synaptic responses to random presynaptic AP trains}

The functions of the mean responses to random presynaptic AP trains against the mean presynaptic AP rate are quite similar to ones of the steady state values for the regular presynaptic AP trains (Fig. 10 and Figs. 2-5(D)). The qualitative similarity indicates the mean presynaptic AP rates are one of the most important factors for synaptic responses to be qualitatively characterized.

\subsubsection{Tuning curve}

In the previous section, the mean response has been analyzed as a function against the mean rate of random AP trains (Fig. 10). In the case of a random presynaptic AP train, however, the train involves various ISIs and synaptic responses vary due to the ISIs. To analyze synaptic responses to individual ISIs in an ongoing presynaptic AP train and to understand more details of the natures in the four types of synapses, the tuning curve is employed in this section (Fig. 11). The tuning curve 
A

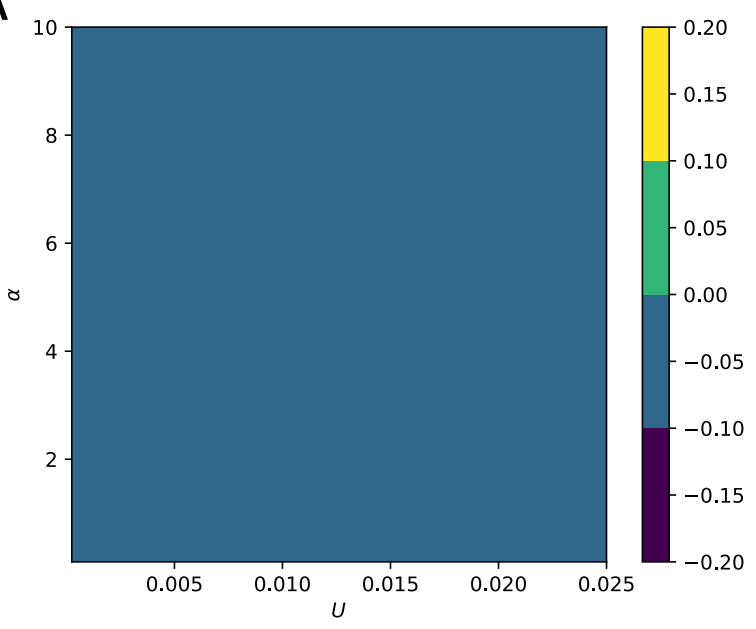

C

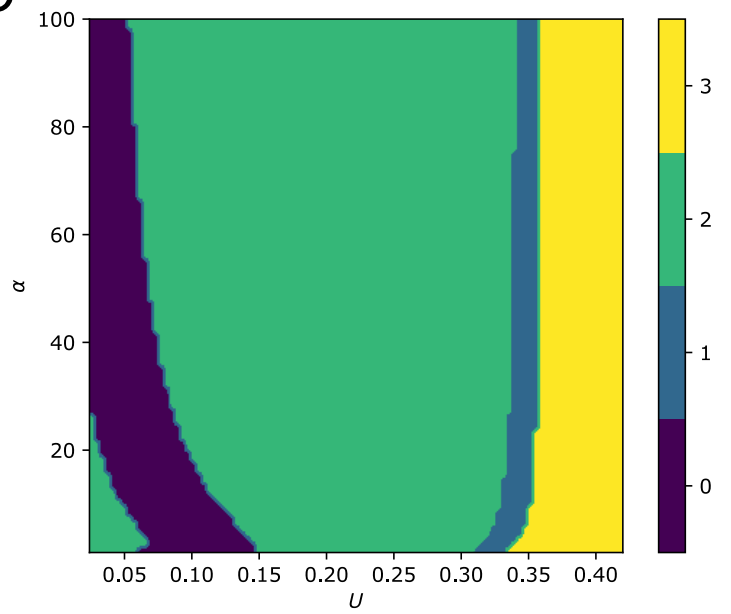

B

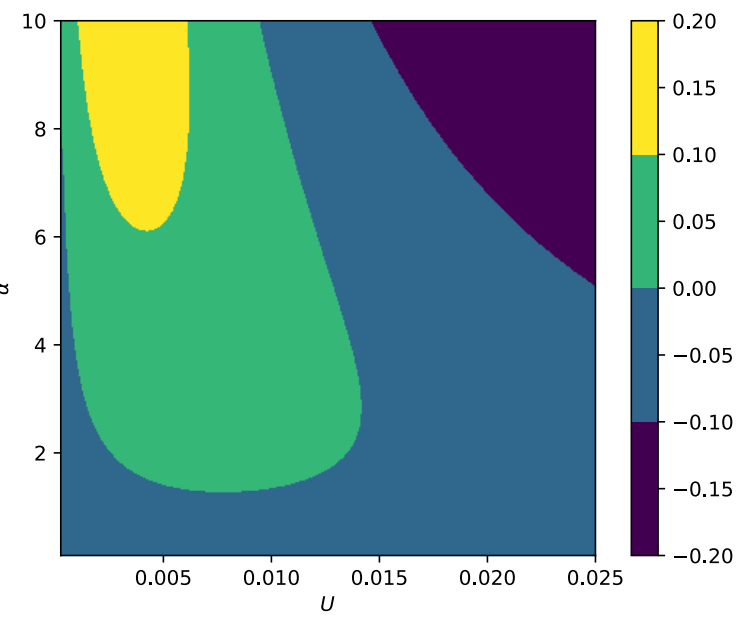

D

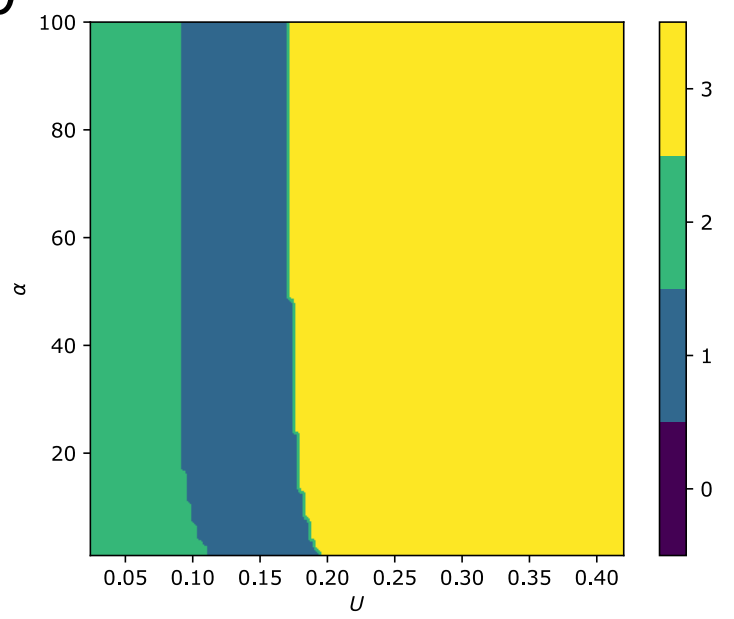

Fig. 8. Dependency of parameters $U$ and $\alpha$ on FG and FD. The parameter dependency on FG when (A) $10 \mathrm{sp} / \mathrm{s}$ and (B) $40 \mathrm{sp} / \mathrm{s}$ regular AP trains are applied. The colors indicate values of the fitting parameter $p_{1}$. A positive value of $p_{1}$ classifies synaptic responses into FG. (C)(D) The same as (A)(B) but for FD. The color indicates the modes of synaptic responses. Only modes 1 and 3 are regarded as FD. All the parameters of the STP model without $U$ and $\alpha$ are the same as Figs 4 and 5.

is a relationship between a synaptic response and an instantaneous ISI between a presynaptic AP evoking the response and its previous AP.

In the case of $\lambda=10 \mathrm{sp} / \mathrm{s}$, the small ISIs around $20 \mathrm{~ms}$ weakly depress but the larger ISIs strongly depress the synaptic responses of the depression synapse (Fig. 11(A) filled points). The synaptic responses of $\lambda=40 \mathrm{sp} / \mathrm{s}$ inherit this trend although the tuning curve is shrunk by $1 / 4$ in the horizontal axis (Fig. 11(A) open points). The difference between 10 and $40 \mathrm{sp} / \mathrm{s}$ is the absolute value of synaptic responses. Taking into account the first response of the synapse is $0.5 \mathrm{mV}$, the presynaptic AP train with $\lambda=40 \mathrm{sp} / \mathrm{s}$ more depress synaptic responses, compared with that with the mean rate of $\lambda=10 \mathrm{sp} / \mathrm{s}$.

Responses of the facilitation synapse exhibit more facilitation to the smaller ISIs of the presynaptic AP train with $\lambda=10 \mathrm{sp} / \mathrm{s}$ (Fig. 11(B) filled points). The larger ISIs have fewer effects to facilitate the synaptic responses and the ISIs larger than $500 \mathrm{~ms}$ have no longer the facilitation effects on the synaptic responses. In the case of $\lambda=40 \mathrm{sp} / \mathrm{s}$, any ISIs induce the facilitation in synaptic responses of the facilitation synapse (Fig. 11(B) open points). Although the synaptic responses are always facilitated, their effects decrease as the ISIs are larger. In addition, the tuning curve of $40 \mathrm{sp} / \mathrm{s}$ locates above that of $10 \mathrm{sp} / \mathrm{s}$, indicating the synapse favors the higher rate presynaptic AP trains. 


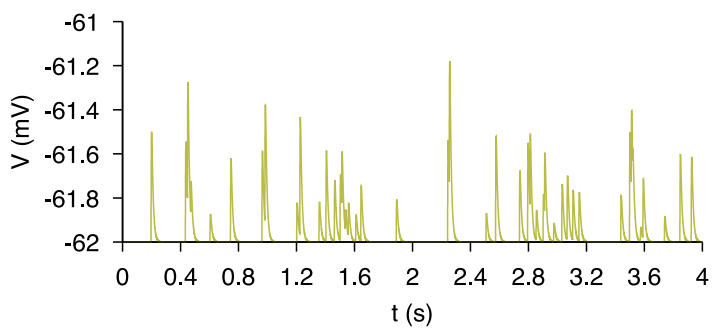

B

| |||||||||||||||||||||||||||||||||||| $\mid$

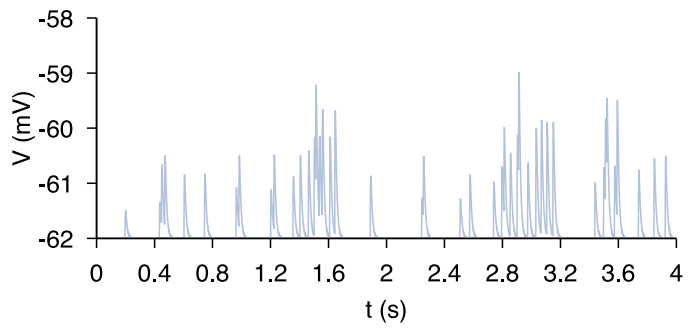

\section{C}

| ||||||||||||||||$\quad|\quad| \quad||||||||||||||||||||$

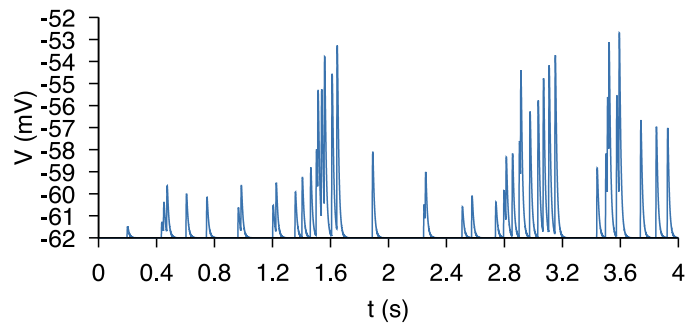

D
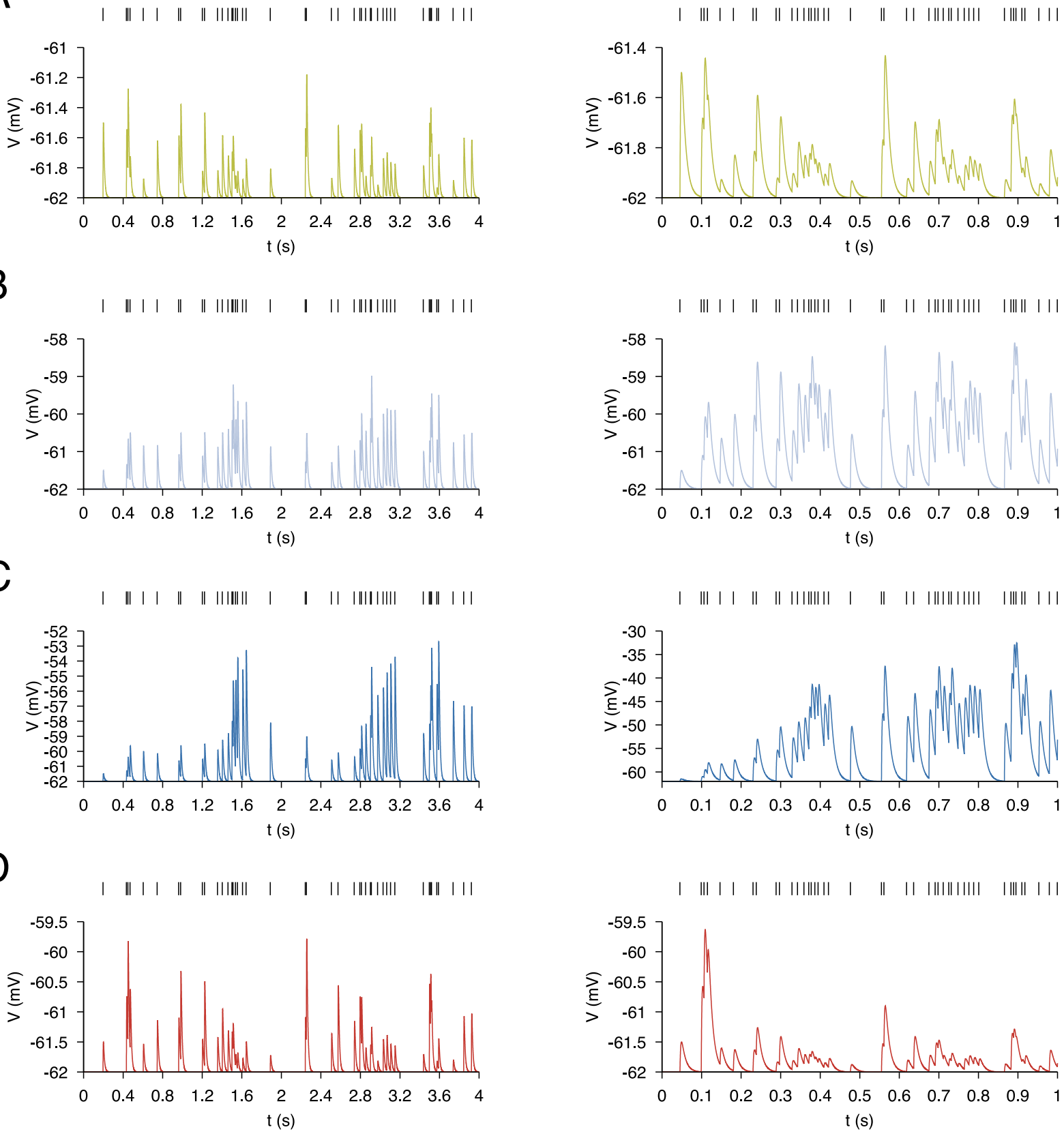

Fig. 9. Responses of (A) depression synapse, (B) facilitation synapse, (C) FG synapse, and (D) FD synapse for the same random presynaptic AP trains (black vertical bars in each panel). All parameters of each synapse are same as Figs. 2-5. The left and the right pannel are cases of 10 and $40 \mathrm{~Hz}$ mean rate of presynaptic AP trains.

The FG synapse exhibits a similar trend with the facilitation synapse (Fig. 11(B), (C)). For the $\lambda=10 \mathrm{sp} / \mathrm{s}$ presynaptic AP train, the responses of the FG synapse is twice as large as that of the facilitation synapse for the ISIs smaller than $300 \mathrm{~ms}$, whereas there are no differences between the responses of the facilitation and the FD synapse for the ISIs larger than $300 \mathrm{~ms}$. Compared with the facilitation synapse, in the case of the FG synapse, there are a much larger gap between the tuning curve of $\lambda=10$ and $40 \mathrm{sp} / \mathrm{s}$ presynaptic AP train. These facts tell us the FG synapse is more sensitive to and is more affected by the presynaptic AP rate than the facilitation synapse.

In the case of $\lambda=10 \mathrm{sp} / \mathrm{s}$, The tuning curve of the FD synapse monotonically decreases as the larger ISIs, which is the same property with the facilitation and the FG synapse (Fig. 11(D)). The different things of the FD synapse from these synapses are the following: the responses of the FD 

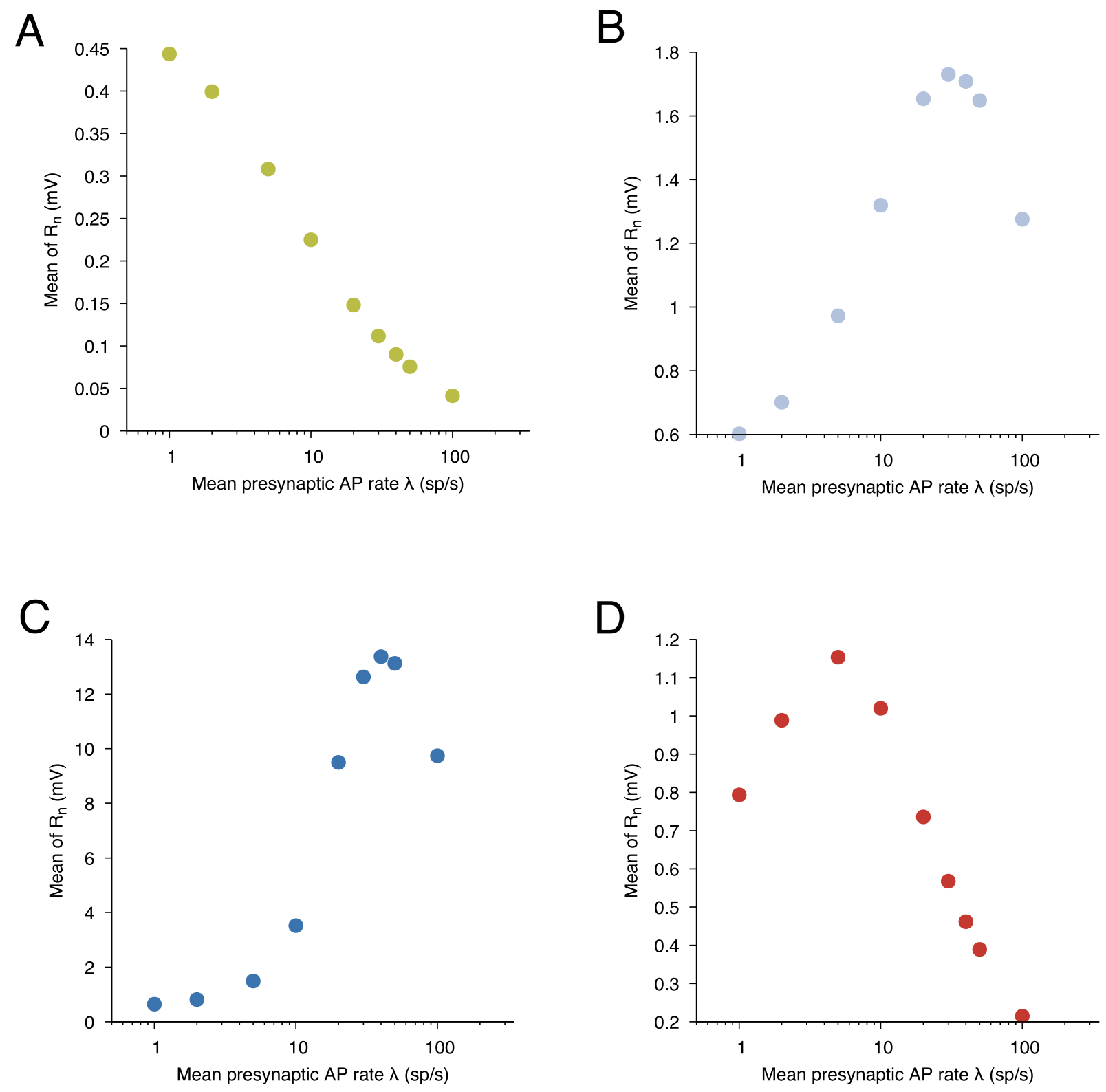

Fig. 10. The mean synaptic responses of (A) depression synapse, (B) facilitation synapse, (C) FG synapse, and (D) FD synapse.

synapse exhibit the facilitation when the ISIs are smaller than $500 \mathrm{~ms}$ and the synaptic responses are switched to the depression for the larger ISIs. Additionally, the tuning curve of the smaller $\lambda$ locates below that of the larger $\lambda$ and is almost flat against the ISIs. These are the shared properties with the depression synapse. Therefore, it can also be seen in the tuning curve that the FD synapse has the characteristics of both the depression and the facilitation synapse.

\subsubsection{Information-theoretic analysis}

The analysis with the turning curve has revealed the trend of synaptic responses to instantaneous ISIs in an ongoing random presynaptic AP train in each type of the four synapses. Namely, the relation between the amplitude of synaptic responses to instantaneous ISIs has been evaluated. To evaluate the amount of the information of the instantaneous ISIs in the synaptic responses rather than the amplitude, the information-theoretic analysis is conducted in this section.

In the case of the depression synapse, the information increases until the presynaptic AP rate reaches $\lambda=1.5 \mathrm{sp} / \mathrm{s}$ (Fig. 12 yellow line). For larger $\lambda$, once the information decreases slightly until $7 \mathrm{sp} / \mathrm{s}$ and increases and converges to around $0.4 \mathrm{sp} / \mathrm{s}$ in the depression synapse. 

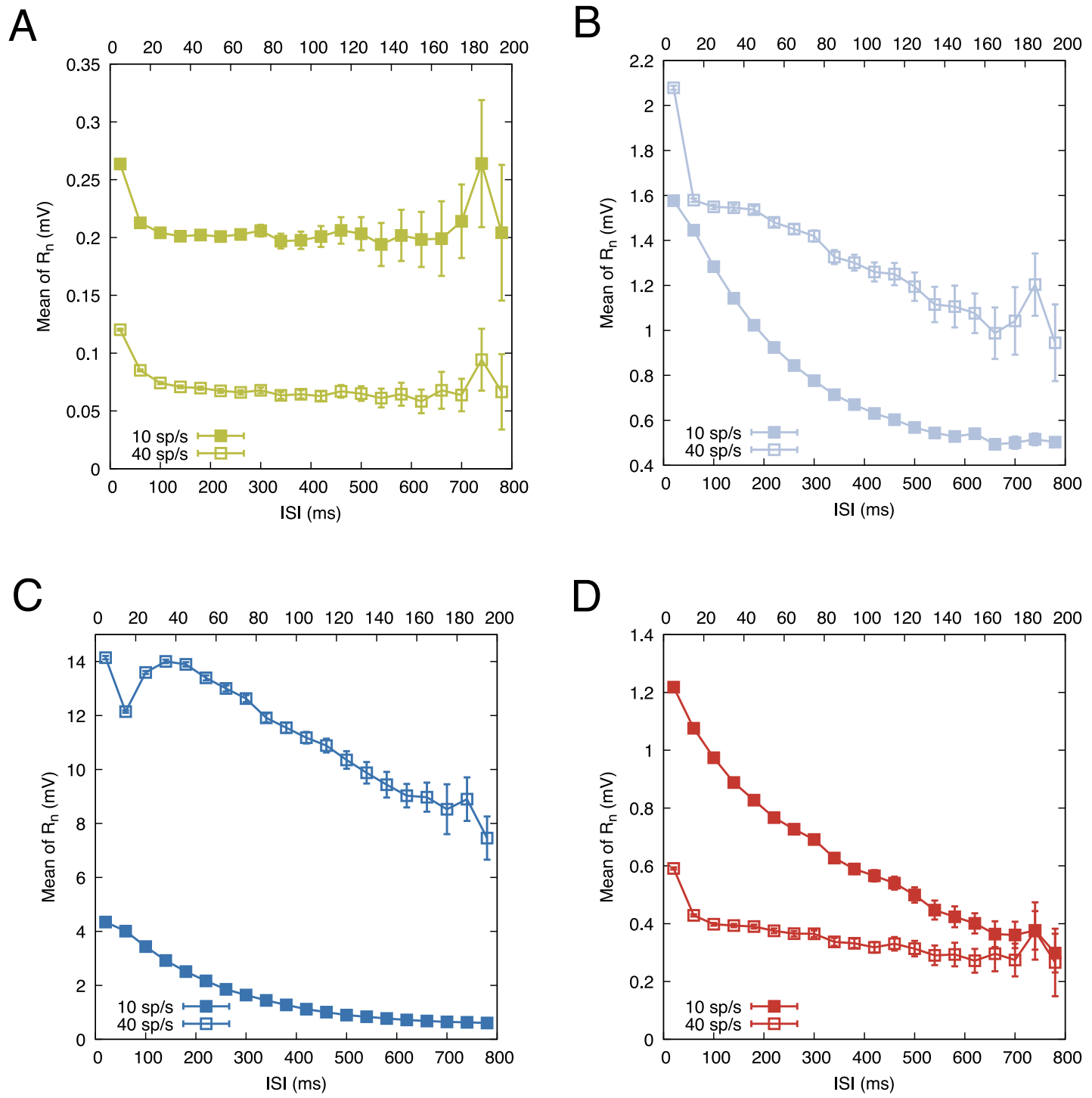

Fig. 11. Tuning curves of the four types of synapses. (A) Depression synapse, (B) facilitation synapse, (C) FG synapse, and (D) FD synapse. In each panel, filled and open squares are cases of 10 and $40 \mathrm{~Hz}$ mean rate of presynaptic APs. The lower- and the upper-horizontal axis are for 10 and $40 \mathrm{~Hz}$ of the mean presynaptic AP rate. The error bars are the $95 \%$ confidence intervals.

Different from the depression synapse, there is an apparent peak in the information of the facilitation synapse against the mean presynaptic AP rate (Fig. 12 light blue line). The peak value of the information is about 2.26 bits. The existence of a peak in the information function is expected because the effects of the STP disappear in small $\lambda$ because small $\lambda$ leads large ISIs which allow all the variables to recover to the baselines. In contrast, large $\lambda$ leads small ISIs and synaptic responses are strongly facilitated. Therefore, the optimal mean presynaptic AP rate $\left(\lambda_{\text {opt }}\right)$ might exist at the mediate level of the mean presynaptic AP rate.

In the case of the FG synapse, $\lambda_{\text {opt }}$ is the same as the one of the facilitation synapse and the function of the information is almost the same in $0 \leq \lambda \leq 5 \mathrm{sp} / \mathrm{s}$ (Fig. 12 blue line). In larger $\lambda$, the information of the FG synapse is smaller than that of the facilitation synapse.

The information function in the FD synapse exhibits a similar trend with the functions in the facilitation and the FG synapses (Fig. 12 red line). However, $\lambda_{\text {opt }}$ is slightly larger than the other synapses and is $3 \mathrm{sp} / \mathrm{s}$. 


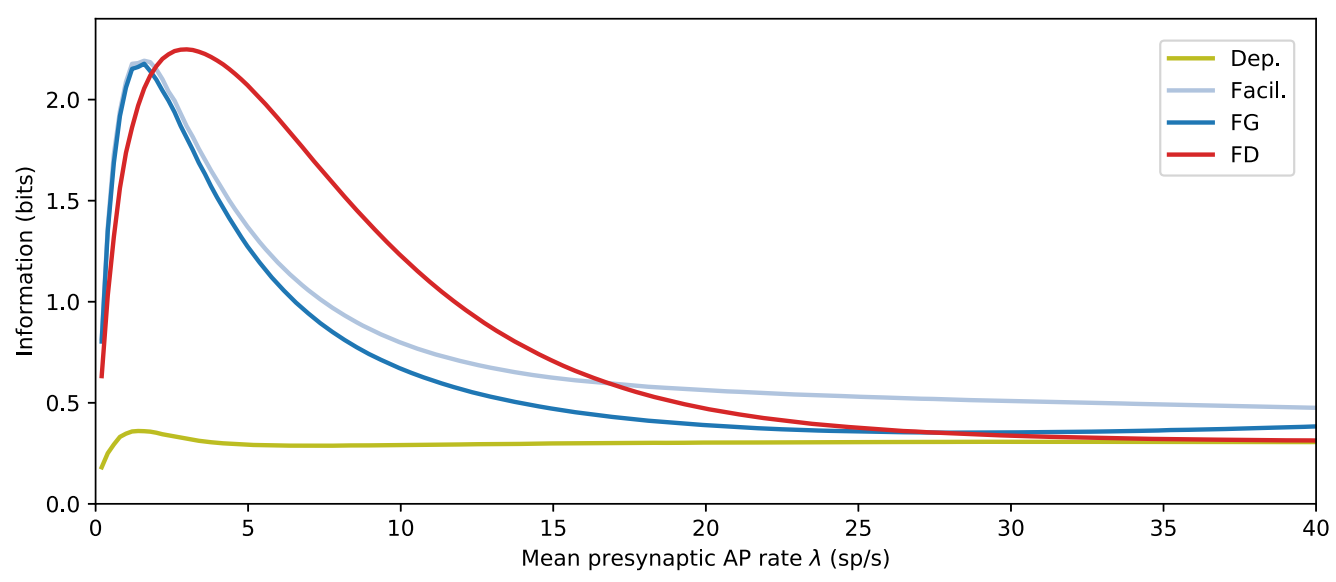

Fig. 12. Information-theoretic analysis. Information contained in postsynaptic responses of the leaky-integrator for given presynaptic AP trains via four types of synapses.

\section{Conclusions and discussions}

In this paper, the augmentation of a canonical phenomenological model of short-term plasticity (STP) has been proposed by introducing the nonlinearity to reproduce postsynaptic responses observed in some target-specific synapses such as facilitation growth (FG) and facilitation-depression (FD), which are not reproducible in the canonical model. As a result, the introduction of the nonlinearity leads the model to the success to trace the FG and the FD. Besides, the augmented model is designed as its computational efficiency is almost the same as the canonical model, indicating the augmented model is usable in large-scale neural network simulations. The choice of the nonlinear function contributes to the computational efficiency of the augmented model.

The mechanisms of short-term depression and facilitation are widely and intensively analyzed in empirical and theoretical studies but are still unclear. Some theoretical studies with numerical simulations suggest several hypotheses of the mechanisms [9]. While there are some hypotheses of the depression and the facilitation, a common key factor is calcium in a presynaptic bouton. In particular, sensors of residual calcium, calcium-channel facilitation, and calcium-buffer saturation are discussed as the candidates of the facilitation mechanisms, and these factors can contribute to the facilitation not only individually but also multiply. Due to these factors or the cooperation of these factors, the neurotransmitter-release probability might complicatedly change and might prominently accelerate the increase and the decrease of the release probability, which is focused on and is expressed by the nonlinearity of the augmented STP model in the current study. Even though the nonlinearity in the augmented model derives from the suggested factors or their combinations, they are still under discussion and the mechanisms of the depression and the facilitation are not clarified yet. However, the nonlinearity introduced in the current study works well in the augmented model that can well trace or reproduce the FG and the FD behaviors. This fact lets the author believe the nonlinearity is the simplified form of the factors or their combinations so that introducing the nonlinearity leads the canonical phenomenological model to succeed in the reproductions of the synaptic responses such as the FG and the FD that are never observed in the canonical phenomenological model.

\section{Acknowledgments}

The research of HK was partially supported by Grant-in-Aid for Young Scientists (B) (No.16K16127) and Grant-in-Aid for Scientific Research (A) (No.20H00596) from JSPS. Part of this work was carried out under the Cooperative Research Project Program of the Research Institute of Electrical Communication, Tohoku University. 


\section{References}

[1] M.A. Castro-Alamancos and B.W. Connors, "Cellular mechanisms of the augmenting response: Short-term plasticity in a thalamocortical pathway," Journal of Neuroscience, vol. 16, no. 23, pp. 7742-7756, 1996.

[2] M.A. Castro-Alamancos and B.W. Connors, "Spatiotemporal properties of short-term plasticity in sensorimotor thalamocortical pathways of the rat," Journal of Neuroscience, vol. 16, no. 8, pp. 2767-2779, 1996.

[3] M.A. Castro-Alamancos, "Short-term plasticity in thalamocortical pathways: Cellular mechanisms and functional roles," Reviews in the Neurosciences, vol. 8, no. 2, pp. 95-116, 1997.

[4] M.V. Tsodyks and H. Markram, "The neural code between neocortical pyramidal neurons depends on neurotransmitter release probability," Proceedings of the National Academy of Sciences of the United States of America, vol. 94, no. 2, pp. 719-723, 1997.

[5] H. Markram., Y. Wang, and M. Tsodyks, "Differential signaling via the same axon of neocortical pyramidal neurons," Proceedings of the National Academy of Sciences of the United States of America, vol. 95, no. 9, pp. 5323-5328, 1998.

[6] C.C.H. Petersen, "Short-term dynamics of synaptic transmission within the excitatory neuronal network of rat layer 4 barrel cortex," Journal of Neurophysiology, vol. 87, no. 6, pp. 2904-2914, 2002.

[7] R.S. Zucker, "Calcium- and activity-dependent synaptic plasticity," Current Opinion in Neurobiology, vol. 9, pp. 305-313, 1999.

[8] R.S. Zucker and W.G. Regehr, "Short-term synaptic plasticity," Annual Review of Physiology, vol. 64 , pp. 355-405, 2002.

[9] W.G. Regehr, "Short-term presynaptic plasticity," Cold Spring Harbor Perspectives in Biology, vol. 4, no. 7, pp. 1-19, 2012.

[10] D. Sussillo, T. Toyoizumi, and W. Maass, "Self-tuning of neural circuits through short-term synaptic plasticity," Journal of Neurophysiology, vol. 97, no. 6, pp. 4079-4095, 2007.

[11] D.V. Buonomano and W. Maass, "State-dependent computations: Spatiotemporal processing in cortical networks," Nature Reviews Neuroscience, vol. 10, no. 2, pp. 113-125, 2009.

[12] I. Hayut, E.E. Fanselow, B.W. Connors, and D. Golomb, "LTS and FS inhibitory interneurons, short-term synaptic plasticity, and cortical circuit dynamics," PLoS Computational Biology, vol. 7, no. 10, 2011.

[13] G. Fuhrmann, I. Segev, H. Markram, and M. Tsodyks, "Coding of temporal information by activity-dependent synapses," Journal of Neurophysiology, vol. 87, no. 1, pp. 140-148, 2002.

[14] B. Szatmáry and E.M. Izhikevich, "Spike-timing theory of working memory," PLoS Computational Biology, vol. 6, no. 8, 2010.

[15] L.F. Abbott and W.G. Regehr, "Synaptic computation," Nature, vol. 431, no. 7010, pp. 796-803, 2004.

[16] J.S. Dittman, A.C. Kreitzer, and W.G. Regehr, "Interplay between facilitation, depression, and residual calcium at three presynaptic terminals," Journal of Neuroscience, vol. 20, no. 4, pp. $1374-1385,2000$.

[17] L.F. Abbott, J.A. Varela, K. Sen, and S.B. Nelson, "Synaptic depression and cortical gain control," Science, vol. 275, no. 5297, pp. 221-224, 1997.

[18] D.L. Cook, P.C. Schwindt, L.A. Grande, and W.J. Spain, "Synaptic depression in the localization of sound," Nature, vol. 421, no. 6918, pp. 66-70, 2003.

[19] M.S. Goldman, P. Maldonado, and L.F. Abbott, "Redundancy reduction and sustained firing with stochastic depressing synapses," Journal of Neuroscience, vol. 22, no. 2, pp. 584-591, 2002.

[20] Z. Rotman, P.Y. Deng, and V.A. Klyachko, "Short-term plasticity optimizes synaptic information transmission," Journal of Neuroscience, vol. 31, no. 41, pp. 14800-14809, 2011.

[21] J.P. Pfister, P. Dayan, and M. Lengyel, "Synapses with short-term plasticity are optimal estimators of presynaptic membrane potentials," Nature Neuroscience, vol. 13, no. 10, pp. 1271-1275, 2010. 
[22] G. Mongillo, O. Barak, and M. Tsodyks, "Synaptic theory of working memory," Science, vol. 319 , no. 5869, pp. 1543-1546, 2008.

[23] M. Beierlein, J.R. Gibson, and B.W. Connors, "Two dynamically distinct inhibitory networks in layer 4 of the neocortex," Journal of Neurophysiology, vol. 90, no. 5, pp. 2987-3000, 2006.

[24] M. Díaz-Quesada, F.J. Martini, G. Ferrati, I. Bureau, and M. Maravall, "Diverse thalamocortical short-term plasticity elicited by ongoing stimulation," Journal of Neuroscience, vol. 34, no. 2, pp. 515-526, 2014.

[25] W. Maass and H. Markram, "Synapses as dynamic memory buffers," Neural Networks, vol. 15, no. 2, pp. 155-161, 2002.

[26] Y.G. Tang, T. Schlumpberger, T.S. Kim, M. Lueker, and R.S. Zucker, "Effects of mobile buffers on facilitation: Experimental and computational studies," Biophysical Journal, vol. 78, no. 6, pp. 2735-2751, 2000.

[27] V. Matveev, A. Sherman, and R.S. Zucket, "New and corrected simulations of synaptic facilitation," Biophysical Journal, vol. 83, no. 3, pp. 1368-1373, 2002.

[28] M.R. Bennett, L. Farnell, and W.G. Gibson, "The facilitated probability of quantal secretion within an array of calcium channels of an active zone at the amphibian neuromuscular junction," Biophysical Journal, vol. 86, no. 5, pp. 2674-2690, 2004.

[29] V. Matveev, R.S. Zucker, and A. Sherman, "Facilitation through buffer saturation: Constraints on endogenous buffering properties," Biophysical Journal, vol. 86, no. 5, pp. 2691-2709, 2004.

[30] A.G. Millar, R.S. Zucker, G.C.R. Ellis-Davies, M.P. Charlton, and H.L. Atwood, "Calcium sensitivity of neurotransmitter release differs at phasic and tonic synapses," Journal of Neuroscience, vol. 25, no. 12, pp. 3113-3125, 2005.

[31] V. Matveev, R. Bertram, and A. Sherman, "Residual bound $\mathrm{Ca}^{2+}$ can account for the effects of $\mathrm{Ca}^{2+}$ buffers on synaptic facilitation," Journal of Neurophysiology, vol. 96, no. 6, pp. 3389-3397, 2006.

[32] B. Pan and R.S. Zucker, "A general model of synaptic transmission and short-term plasticity," Neuron, vol. 62, no. 4, pp. 539-554, 2009.

[33] W.R. Softky and C. Koch, "The highly irregular firing of cortical cells is inconsistent with temporal integration of random EPSPs," Journal of Neuroscience, vol. 13, no. 1, pp. 334-350, 1993.

[34] A.L. Barth and J.F.A. Poulet, "Experimental evidence for sparse firing in the neocortex," Trends in Neurosciences, vol. 35, no. 6, pp. 345-355, 2012. 